\title{
Time experience in social isolation: a longitudinal study during the first months of COVID-19 pandemic in Brazil
}

André Mascioli Cravo ${ }^{1}$, Gustavo Brito de Azevedo ${ }^{1}$, Cristiano Moraes Bilacchi Azarias ${ }^{1}$, Louise

Catheryne Barne ${ }^{2}$, Fernanda Dantas Bueno ${ }^{1}$, Raphael Y. de Camargo ${ }^{1}$, Vanessa Carneiro Morita ${ }^{3}$, Esaú Sirius Ventura Pupo ${ }^{1}$, Renan Schiavolin Recio ${ }^{4}$, Mateus Silvestrin ${ }^{1}$, Raymundo Machado de Azevedo Neto 5 .

${ }^{1}$ Universidade Federal do ABC (UFABC) - Centro de Matemática, Computação e Cognição, BR

${ }^{2}$ Département Traitement de l'Information et Systèmes, ONERA, Salon-de-Provence, FR

${ }^{3}$ Institut de Neurosciences de la Timone, UMR7289, CNRS, Aix-Marseille Université, F13005 Marseille, FR

${ }^{4}$ NeurUX - Soluções em Neurociência e Tecnologia Ltda, BR

${ }^{5}$ Instituto do Cérebro, IIEP-HIAE, BR

Acknowledgements:We thank Marc Wittmann and the Timing and Cognition Lab for insightful comments on earlier versions of the manuscript. AMC was supported by grant \#2017/25161-8, São Paulo Research Foundation (FAPESP). GBA was supported by grant \#2019/25572-3, São Paulo Research Foundation (FAPESP). FDB was supported by grant \#2017/24575-3, São Paulo Research Foundation (FAPESP). ESVP was supported by grant \#2019/06423-7, São Paulo Research Foundation (FAPESP). MS was supported by grant \#2016/24951-2, São Paulo Research Foundation (FAPESP). 


\section{Abstract}

Social distancing in response to the Covid-19 pandemic brought several modifications in our daily lives. With these changes, some people have reported alterations in their feelings of how fast time was passing. In this study, we assessed whether and how social distancing influenced participants' time awareness and production of time intervals. Participants $(n=3855)$ filled the first questionnaire approximately 60 days after the start of social isolation in Brazil and weekly questionnaires for 15 weeks during social distancing. Our results indicate that time was perceived as expanded at the beginning of social isolation, but this feeling decreased across the weeks. Time awareness was strongly associated with psychological factors such as loneliness, stress, and positive emotions, but not with time production. This relation was shown between participants and within their longitudinal reports. Taken together, our findings show how emotions are a crucial aspect of how time is felt. 


\section{Introduction}

In 2020 the world faced an unprecedented health crisis with the outbreak of the Sars-CoV-2 pandemic. High transmission, morbidity, and mortality rates forced governments worldwide to implement social distancing measures to prevent the virus's spread. As prolonged periods of social distancing were enforced in some parts of the world, people started reporting changes in their mental health/status, including changes in subjective time (Wittmann, 2020). What is commonly referred to as subjective time or temporal experience involves multidimensional and interdependent abilities such as: time perspective, which is the conceptualization of a past, a present, and a future; time-estimation, related to the measurement of clock time; and time awareness, which is the subjective impression of time as moving quickly or slowly (Locsin, 1993; Wittmann \& Lehnhoff, 2005).

Temporal experience is not immune to contextual and individual factors and is modulated by several factors, such as boredom, impulsivity, anxiety, routine, and emotions (Droit-Volet et al., 2013; Wittmann, 2009). Not surprisingly, it has been shown that subjective time is also affected in isolation-based experiments (e.g., people isolated in bunkers or caves) (Crawford \& Thor, 1967; Siffre, 1964; Thor \& Crawford, 1964). One key aspect of isolation-based experiments is that they are based on the radical removal of any time markers in the environment (e.g., clocks and sunlight). Social isolation resulting from COVID-19 containment measures, on the other hand, required people to be isolated at their homes while still having access to time markers. This peculiar situation allows for a more nuanced investigation of factors contributing to distortions in time estimation and time awareness.

So far, studies that investigated time perception during COVID-19 social isolation showed a mixed pattern of results. Three studies, two performed in France and one in Italy, have shown that people mainly reported the sensation that time was passing more slowly than usual (Cellini et al., 2020; Droit-Volet et al., 2020; Martinelli et al., 2020). On the other hand, two studies in the UK found that an equal number of participants reported that the previous day had passed more slowly or quickly, suggesting a more complex relationship between the awareness of time and social isolation (R. Ogden, 2021; R. S. Ogden, 2020). The main factors associated with the sensation of time slowing down across these studies were: high levels of 
stress, boredom and sadness, low levels of happiness, depression, young age, and dissatisfaction with social interactions (Cellini et al., 2020; Droit-Volet et al., 2020; Martinelli et al., 2020; R. Ogden, 2021; R. S. Ogden, 2020). These studies, however, have three main limitations: (1) asking a single question to characterize time awareness instead of multi-item scales; (2) no assessment of other dimensions of time experience such as time-estimation; (3) a single assessment during the first weeks of the social isolation instead of a prolonged, longitudinal, period of social isolation.

Here, we aim to characterize temporal experience during social isolation in more detail. To assess time perception, we used both an interval production task and subjective time awareness scales. Critically, we used scales that measured two different aspects of time awareness: time expansion and time pressure (Wittmann \& Lehnhoff, 2005). These two scales measure distinct features of time awareness, namely, the subjective pace of time (time expansion) and time pressure in daily and life goals (time pressure). Although these measures can sometimes be negatively correlated, they measure different aspects of our temporal experience. For instance, a patient with a terminal disease can perceive more time pressure while still feeling a slower pace of time. These different aspects of time perception allow for a more comprehensive investigation of the subjective feelings associated with the experience of time during the COVID-19 pandemic's massive social isolation.

Furthermore, instead of focusing only on the first weeks of social isolation, we present longitudinal data collected weekly for five months. In all sessions, temporal reports were combined with measures of stress, well-being, affect, and other custom-made scales, which were used to investigate which factors were more strongly associated with different aspects of temporal experience. We also collected written reports of participants' experiences during social isolation to explore possible affective biases in the period.

Lastly, our sampled population is from Brazil, a country that suffered from severe rates of cases and deaths due to COVID-19 (as of this writing, Brazil ranks second worldwide in the number of deaths related to the pandemic). The country's serious situation prolonged the necessity of social isolation measures and an emotional toll on its population, which might have a more detectable influence on how people perceive time. Since the government did not 
adopt a rigorous and homogeneous lockdown, it was possible to explore how individually embraced social isolation influenced time perception across this period. 


\section{Results}

We aimed to understand whether and how social isolation influenced participants' time awareness and production of time intervals during the COVID-19 pandemic. To that end, we collected data through an online survey published in social media outlets. A total of 3855 participants completed the full survey. Table 1 describes our sample's demographic information and social isolation situation. Briefly, respondents were adult Brazilians mostly from the southeast region (80.50\%); women (74.32\%); highly educated (college degree, 71.78\%); middle-upper class (33.08\%); and working in the education (19.43\%) or health care (15.36\%) sectors. Although this sample is not representative of the country's population, possibly due to the online survey format and the recruitment process, we have no reason to believe that this bias in our sample would prevent us from addressing our research questions. Most participants reported being socially isolated for a period of 51 days when participating in the first session (median $=51$, range from 0 to 120 days) at their own houses (90.56\%) when they completed the first session.

To investigate whether and how time awareness changed throughout the weeks of social isolation, participants were further invited to complete weekly sessions. This questionnaire had a similar structure to that of the first session (more details below), without the demographic questions. Participants were informed that they did not have to fill the questionnaire every week but were asked to participate as much as possible. Weekly sessions lasted until August 6th, 14 weeks after the beginning of this study. Compared to the first session, there was a moderate dropout of participants: out of the 3855 participants that took part in the first session, 1975 returned to complete at least one more weekly session. We had a steady decrease in the number of responses throughout the weeks (Figure 1). For the weekly sessions, we focused on data from participants that completed at least 4 weekly sessions (25\% of the sessions), for a total of 953 participants and 7497 responses. 


\begin{tabular}{|c|c|c|c|c|c|}
\hline \multicolumn{6}{|c|}{ Participants } \\
\hline & MIN & Q1/4 & Q2/4 & Q3/4 & $\operatorname{MAX}$ \\
\hline Age (years) & 18 & 25 & 34 & 47 & 86 \\
\hline Isolation (days) & 0 & 45 & 51 & 60 & 120 \\
\hline \multirow{2}{*}{ Education (\%) } & $<$ Level 1 & $<$ Level 2 & $<$ Level 3 & $<$ College & College \\
\hline & 0.08 & 0.18 & 0.49 & 27.06 & 71.78 \\
\hline \multirow{2}{*}{ Income (\%) } & $0.5 x$ & $1 x$ & $2 x$ & $4 x$ & $>8 x$ \\
\hline & 4.41 & 14.4 & 18.18 & 21.82 & 33.08 \\
\hline \multirow{2}{*}{ Gender (\%) } & She & $\mathrm{He}$ & Other & & \\
\hline & 74.32 & 25.03 & 0.42 & & \\
\hline \multirow{2}{*}{$\begin{array}{c}\text { Residence in Brazil } \\
(\%)\end{array}$} & $\mathbf{s}$ & SE & MW & NE & $\mathbf{N}$ \\
\hline & 7.91 & 80.5 & 2.75 & 7.69 & 1.18 \\
\hline \multicolumn{6}{|c|}{ Company during social isolation } \\
\hline & MIN & Q1/4 & Q2/4 & Q3/4 & MAX \\
\hline People (n) & 0 & 1 & 2 & 3 & 25 \\
\hline Kids (n) & 0 & 0 & 0 & 1 & 10 \\
\hline \multirow{2}{*}{ Pets (\%) } & No & Yes & & & \\
\hline & 43.06 & 56.94 & & & \\
\hline \multicolumn{6}{|c|}{ Housing conditions (areas) during social isolation } \\
\hline & MIN & Q1/4 & Q2/4 & Q3/4 & MAX \\
\hline Indoor $(n)$ & 1 & 5 & 7 & 9 & 25 \\
\hline Outdoor $(n)$ & 0 & 1 & 2 & 3 & 10 \\
\hline \multirow{2}{*}{ Privacy (\%) } & No & Yes & & & \\
\hline & 11.11 & 88.89 & & & \\
\hline \multicolumn{6}{|c|}{$\begin{array}{l}\text { Table 1. Demographic information of participants. Participants' age in years, number of days in } \\
\text { isolation, education levels, personal income based on minimum wage, gender, and residence in } \\
\text { Brazil (S: South, SE: Southeast, MW: Midwest, NE: Northeast, N: North) are described in the upper } \\
\text { panel. The middle panel illustrates participants' company during social isolation: number of people, } \\
\text { number of kids, and whether they had pets. The bottom panel describes their housing condition } \\
\text { during social isolation: number of indoor areas, number of outdoor areas, and whether they had a } \\
\text { place to be on their own to have some privacy. }\end{array}$} \\
\hline
\end{tabular}



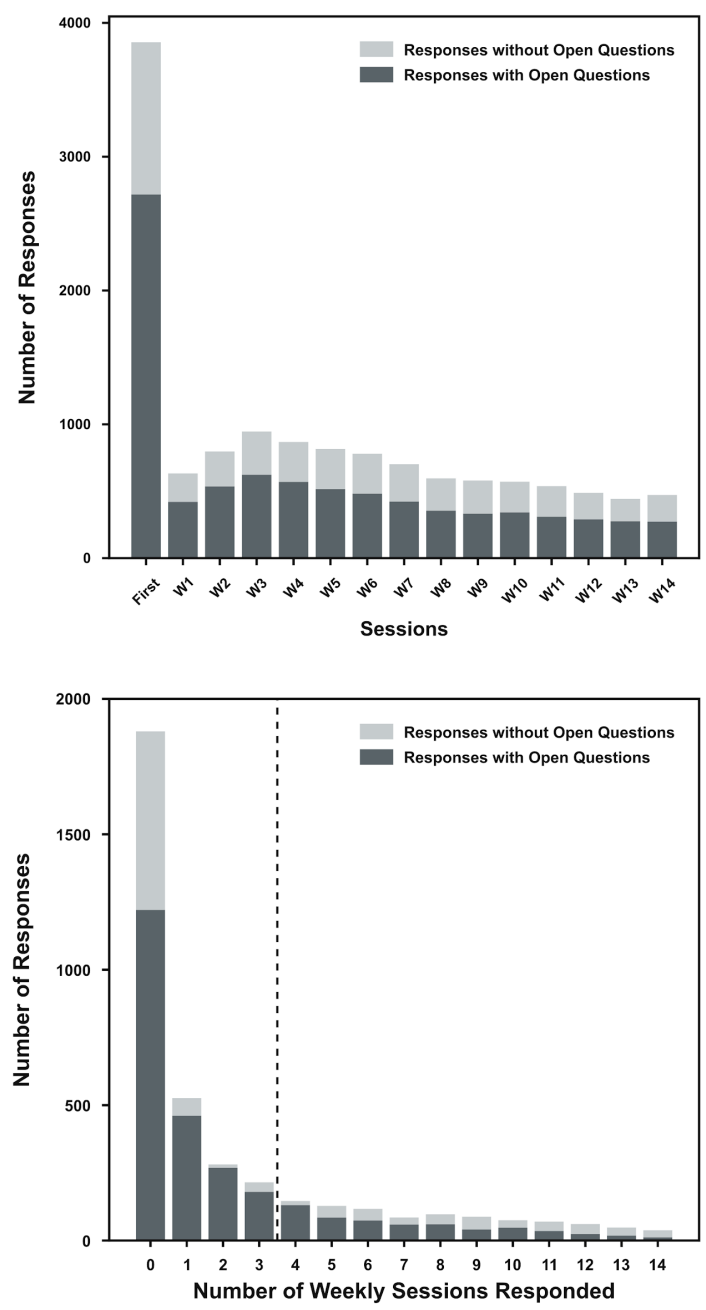

Figure 1. Number of participants and responses. Each bar on the upper panel shows the number of responses in each weekly session. On the lower panel, each bar indicates the number of respondents who completed the correspondent number of weekly sessions - zero indicates completing the first session only. The vertical dashed line indicates the inclusion criteria for weekly session analysis (participants that responded more than three times). In both panels, the dark-filled bars represent full responses (questionnaire and open questions), whereas light-filled bars represent completing the survey but not the optional open-questions at the end. 


\section{Time awareness during social isolation}

\section{$\underline{\text { First session }}$}

Time awareness (the subjective impression of time as moving quickly or slowly) was measured using two scales (Wittmann \& Lehnhoff, 2005): one that measured the feeling of time pressure (time feeling scarce) and the other that measured the feeling of time expansion (time pace slow). Given that these scales were used for the first time in Brazilian Portuguese, we also investigated their construct validity with factor analysis (more details in the methods session).

In a first analysis, we compared time awareness during social isolation with time awareness before the pandemic (Figure 2). Participants presented strong changes in time awareness during social isolation, with an increase of temporal expansion for aproximatly $65 \%$ of respondents [before isolation: median (interquartile range-IQR) $=24.72$ (8.85 to 43.16), during isolation: median $(\mathrm{IQR})=43.2$ (18.46 to 66.2), both scales measured on a range from 0-100; Wilcoxon signed rank test: $\mathrm{W}$-val $=1445051, \mathrm{p}<0.001$, Common Language Effect Size ${ }^{1}$ $($ CLES $)=0.347$ ] and a decrease of temporal pressure for aproximatly $75 \%$ of them [before isolation: median $(\mathrm{IQR})=69.40$ (51.28 to 84.87$)$, during isolation: median $(\mathrm{IQR})=40.08(20.16$ to 60.21); Wilcoxon signed rank test: $\mathrm{W}$-val $=903198.5, \mathrm{p}<0.001$, CLES $=0.751]$. There was a small, although significant, negative correlation between both time awareness measures (Shepherd rho $=-0.152, \mathrm{p}<0.05)$.

\footnotetext{
${ }^{1}$ The Common Language Effect size is defined as the probability that a score sampled at random from one distribution will be greater than a score sampled from some other distribution and varies between 0 to 1 (McGraw \& Wong, 1992; Vargha \& Delaney, 2000).
} 

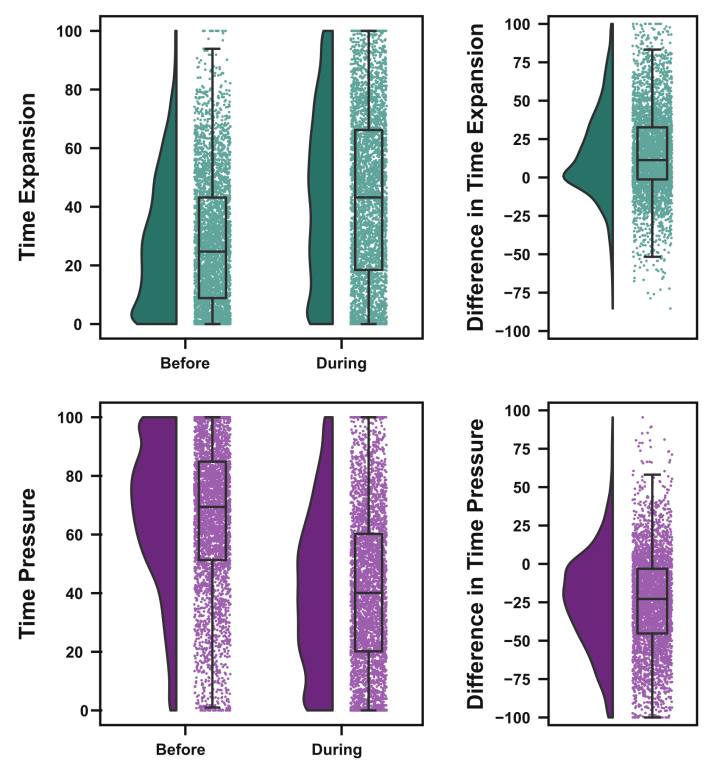

Figure 2. Social isolation modulates time awareness. Time expansion and pressure before and during social isolation, as measured in the first session. Raincloud plots showing group distributions as un-mirrored violin plots (probability density functions), boxplots with medians and interquartile ranges, whiskers with 5th and 95th percentiles, and individual data points (horizontally jittered) (adapted from (Allen et al., 2019). Difference plots were calculated by subtracting the reported time awareness during social isolation from the reported time awareness before social isolation.

Factors associated with distortions in the time awareness during the social isolation

To investigate possible factors that influenced time awareness during social isolation, our survey included scales that measured stress and wellbeing (PSS-10 and WHO-5) and positive and negative affect (PANAS). We further developed questionnaires to assess participants' sense of loneliness, their opinions on the social isolation protocol, changes in their quality of life, and changes in their daily routine. These scales and questionnaires were combined with the demographics described in Table 1 and with questions on whether participants knew someone closely related to them who had been sick due to COVID-19. A complete list of the features used with a brief description of their meanings and descriptive results can be seen in Table 2 . 


\begin{tabular}{|c|c|c|c|}
\hline \multicolumn{4}{|c|}{ Yes-No Questions } \\
\hline & \begin{tabular}{|l} 
Score \\
(Proportion of \\
participants)
\end{tabular} & $\begin{array}{l}\text { Theoretical } \\
\text { Range }\end{array}$ & Meaning \\
\hline $\begin{array}{l}\text { COVID symptoms } \\
\text { myself }\end{array}$ & 0.08 & $0-1$ & $\begin{array}{l}\text { The proportion of participants who presented COVID symptoms } \\
\text { themselves }\end{array}$ \\
\hline $\begin{array}{l}\text { COVID symptoms } \\
\text { family }\end{array}$ & 0.16 & $0-1$ & $\begin{array}{l}\text { The proportion of participants who had someone of their family with } \\
\text { COVID symptoms }\end{array}$ \\
\hline $\begin{array}{l}\text { COVID symptoms } \\
\text { friend }\end{array}$ & 0.18 & $0-1$ & $\begin{array}{l}\text { The proportion of participants who had a friend with COVID } \\
\text { symptoms }\end{array}$ \\
\hline $\begin{array}{l}\text { COVID hospital } \\
\text { myself }\end{array}$ & 0.00 & $0-1$ & The proportion of participants who were hospitalized due to COVID \\
\hline $\begin{array}{l}\text { COVID hospital } \\
\text { family }\end{array}$ & 0.06 & $0-1$ & $\begin{array}{l}\text { The proportion of participants who had someone in their family } \\
\text { hospitalized due to COVID }\end{array}$ \\
\hline $\begin{array}{l}\text { COVID hospital } \\
\text { friend }\end{array}$ & 0.11 & $0-1$ & $\begin{array}{l}\text { The proportion of participants who had a friend hospitalized due to } \\
\text { COVID }\end{array}$ \\
\hline $\begin{array}{l}\text { COVID deaths } \\
\text { family }\end{array}$ & 0.02 & $0-1$ & $\begin{array}{l}\text { The proportion of participants who had a family member who died of } \\
\text { COVID }\end{array}$ \\
\hline $\begin{array}{l}\text { COVID deaths } \\
\text { friend }\end{array}$ & 0.07 & $0-1$ & The proportion of participants who had a friend who died of COVID \\
\hline \multicolumn{4}{|c|}{ Scale Questions } \\
\hline & $\begin{array}{l}\text { Score (Median and } \\
\text { IQR) }\end{array}$ & $\begin{array}{l}\text { Theoretical } \\
\text { Range }\end{array}$ & Meaning \\
\hline Covid Total Score & $0(0-1)$ & {$[0$ 8 8} & Sum of all yes/no COVID responses \\
\hline \multicolumn{4}{|c|}{ Affect (PANAS Scale) } \\
\hline Positive Affect & $23.0(18.0-28.0)$ & {$[945]$} & $\begin{array}{l}\text { Positive emotions (lower/higher values indicate lower/higher positive } \\
\text { emotions) }\end{array}$ \\
\hline Negative Affect & $24.0(18.0-31.0)$ & {$\left[\begin{array}{lll}10 & 50\end{array}\right]$} & $\begin{array}{l}\text { Negative emotions (lower/higher values indicate lower/higher } \\
\text { negative emotions) }\end{array}$ \\
\hline \multicolumn{4}{|c|}{ Stress and Well-Being } \\
\hline PSS & $21.0(16.0-26.0)$ & {$[040]$} & Stress (lower/higher values indicate lower/higher stress levels) \\
\hline WHO & $12.0(8.0-16.0)$ & {$[0$ 25] } & Well being (lower/higher values indicate lower/higher well-being) \\
\hline \multicolumn{4}{|c|}{ Sense of Isolation } \\
\hline Loneliness & $9.0(-1.0-29.0)$ & {$[-50+50]$} & How much participants' feeling of loneliness decreased/increased \\
\hline Distancing & $26.0(11.0-46.0)$ & {$[-50+50]$} & How much participants' feeling of distancing decreased/increased \\
\hline $\begin{array}{l}\text { Personal } \\
\text { Interactions }\end{array}$ & $-10.0(-28.0-13.0)$ & {$[-50+50]$} & How much participants' personal interactions decreased/increased \\
\hline $\begin{array}{l}\text { Work and study } \\
\text { interactions }\end{array}$ & $-13.0(-30.0-8.0)$ & {$[-50+50]$} & How much participants' work/study interactions decreased/increased \\
\hline Personal Care & $8.0(-21.0-27.0)$ & {$[-50+50]$} & $\begin{array}{l}\text { How much participants' time for personal care (e.g. hobbies) } \\
\text { decreased/increased }\end{array}$ \\
\hline \multicolumn{4}{|c|}{ Opinion on social distancing } \\
\hline Opinion on helping & $86.0(68.0-100.0)$ & {$\left[\begin{array}{ll}0 & 100]\end{array}\right.$} & How much participants believe their social distancing is helping \\
\hline Agreement & $95.0(75.0-100.0)$ & {$\left[\begin{array}{ll}0 & 100\end{array}\right]$} & How much participants agree with the social distancing measures \\
\hline News & $78.0(58.0-100.0)$ & {$\left[\begin{array}{ll}0 & 100]\end{array}\right.$} & How much participants were following the news about COVID \\
\hline
\end{tabular}




\begin{tabular}{|c|c|c|c|}
\hline Self Risk & $56.0(33.0-75.0)$ & {$\left[\begin{array}{ll}0 & 100\end{array}\right]$} & $\begin{array}{l}\text { How much participants believe they are in danger of being infected } \\
\text { by COVID }\end{array}$ \\
\hline Others' Risk & $77.0(61.0-99.0)$ & {$\left[\begin{array}{ll}0 & 100\end{array}\right]$} & $\begin{array}{l}\text { How much participants believe loved ones are in danger of being } \\
\text { infected by COVID }\end{array}$ \\
\hline \multicolumn{4}{|c|}{ Quality of Life } \\
\hline Finances & $-8.0(-27.0-1.0)$ & {$[-50+50]$} & How much participants' financial condition worsened/improved \\
\hline $\begin{array}{l}\text { Work and study } \\
\text { quality }\end{array}$ & $-16.0(-33.0-8.0)$ & {$[-50+50]$} & How much participants' work/study conditions worsened/improved \\
\hline Leisure & $-31.0(-49.0--10.0)$ & {$[-50+50]$} & How much participants' leisure worsened/improved \\
\hline Family & $-7.0(-29.0-14.0)$ & {$[-50+50]$} & How much participants' family routine worsened/improved \\
\hline Exercises & $-26.0(-49.0-0.0)$ & {$[-50+50]$} & How much participants' exercises routine worsened/improved \\
\hline \multicolumn{4}{|c|}{ Routine changes } \\
\hline $\begin{array}{l}\text { Hours of work and } \\
\text { study }\end{array}$ & $-30.0(-50.0-0.0)$ & {$[-50+50]$} & $\begin{array}{l}\text { How much participants' hours in their work/study place } \\
\text { decreased/increased }\end{array}$ \\
\hline Hours of leisure & $-49.0(-50.0--35.0)$ & {$[-50+50]$} & $\begin{array}{l}\text { How much participants' hours dedicated to social events (happy } \\
\text { hours, restaurants) decreased/increased }\end{array}$ \\
\hline $\begin{array}{l}\text { Interaction with } \\
\text { housemates }\end{array}$ & $30.0(1.0-50.0)$ & {$[-50+50]$} & $\begin{array}{l}\text { How much participants' hours spent with housemates } \\
\text { decreased/increased }\end{array}$ \\
\hline Hours spent online & $26.0(10.5-43.0)$ & {$[-50+50]$} & $\begin{array}{l}\text { How much time participants spent in online interactions } \\
\text { decreased/increased }\end{array}$ \\
\hline $\begin{array}{l}\text { Hours spent } \\
\text { outside }\end{array}$ & $-50.0(-50.0--37.0)$ & {$[-50+50]$} & $\begin{array}{l}\text { How much participants' hours out in cultural events } \\
\text { decrease/increase }\end{array}$ \\
\hline
\end{tabular}

In a first analysis, pairwise correlations between all measures were estimated across participants (Spearman's rank correlation). To illustrate further the associations between different measures, we used hierarchical cluster analysis to build a dendrogram in which scales that are more strongly associated are placed on branches closer together. As can be seen in Figure 3, there were expected associations within demographic information (e.g., age, scholarity, and income) and within information about the location in which participants were during social isolation (e.g., number of rooms in the house, number of people in the same place). Moreover, there were associations between scales used to measure emotions, stress, and well-being. Critically, time awareness during social isolation seems to be more associated with scales that assess well-being, stress, and emotions. 

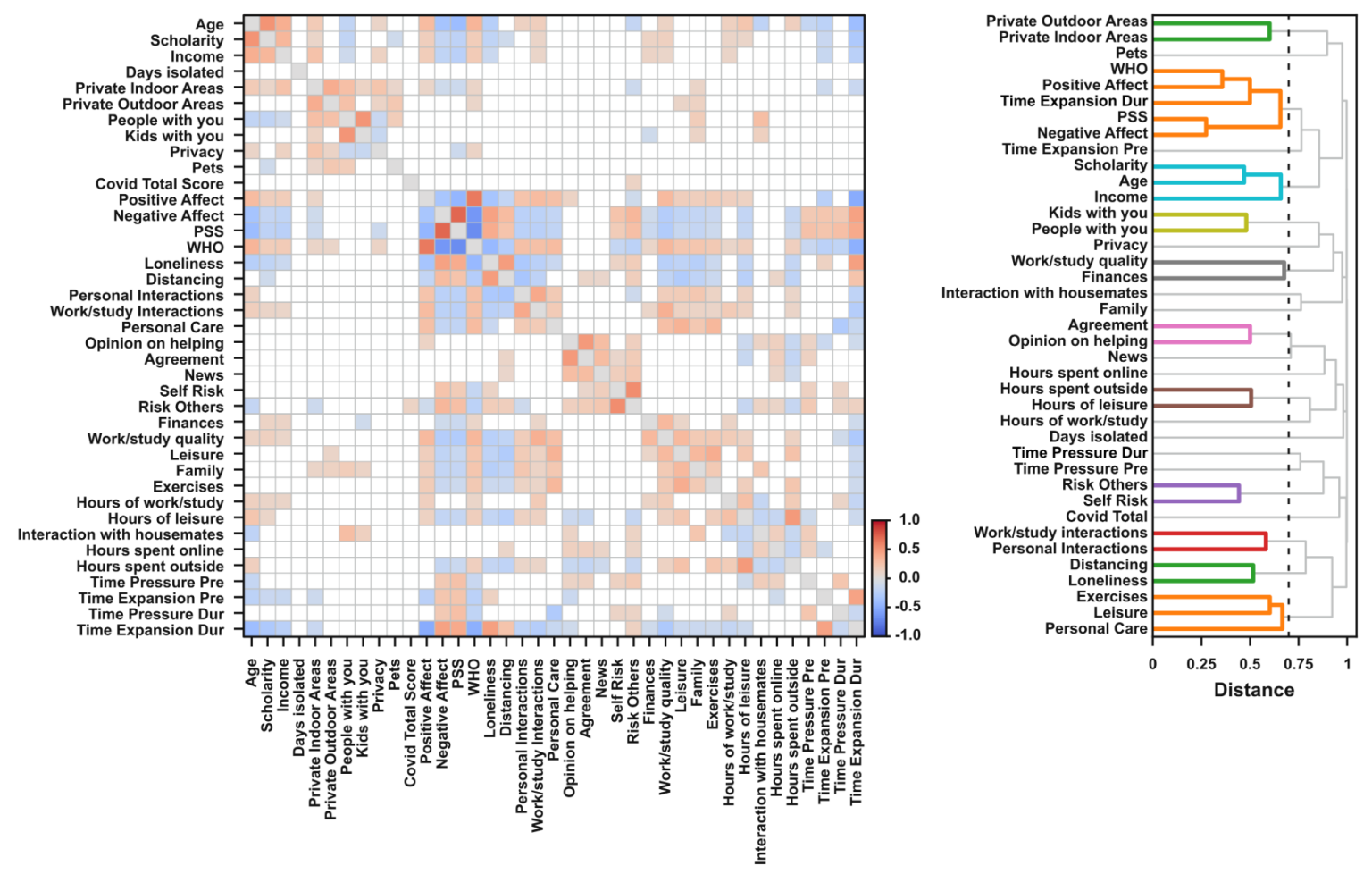

Figure 3. Pairwise correlations between measures and hierarchical cluster analysis in the first session. Left panel shows the pairwise Spearman correlations across measures in the first session. Only effects stronger than 0.1 are presented. The color of individual points indicates the sign and strength of estimated coefficients. Right panel shows the estimated dendrograms using hierarchical clustering analyses of the calculated distances between scales (Distance $=1-\mid$ rho|). The black vertical lines represent the cut threshold, which was defined at a distance between scales with a correlation of medium effect size $(\mid$ rho $\mid=0.3)$. Links connecting nodes with distances smaller than or equal to the threshold are marked as belonging to the same cluster.

We examined which factors were associated with stronger feelings of time expansion and pressure via a gradient boosting regression. Models were fitted on data from the first session using 5-fold cross-validation and fitted 500 times (each using a different 5-fold separation). Model performance was evaluated using three approaches: (1) Estimating the coefficient of determination (R2) between predicted and observed values. This coefficient may range up to 1 for a perfect fit and be zero or even negative (when the mean of the data provides a better fit than the predicted values); (2) Comparing performance with shuffled models, in which the score to be estimated (time pressure or expansion) was shuffled across participants on the training set within each fold, breaking the relationship between the variables of interest 
and the observed scores; (3) Comparing performance with a baseline model, in which only time expansion and time pressure in the period before social isolation were used as predictors.

For both scales of time awareness, we found good predictive scores (measured by the coefficient of determination between predicted and observed values). For time expansion, the full model had a median R2-score of 0.53 , with a minimum R2 across fits of 0.52 and a maximum R2 of 0.54 . The shuffled model for time expansion, as expected, had close to chance values of R2 (median $=-0.07$, $\min =-0.12, \max =-0.03$ ), while the simpler model with only time expansion and time pressure before social isolation had lower R2 values than the complete model $($ median $=0.14, \min =0.12, \max =0.16$ ). A similar pattern was found for time pressure, with good performance for the full model (median $\mathrm{R} 2=0.25, \min =0.23$, $\max =0.27$ ), chance levels for the shuffled model (median $\mathrm{R} 2=-0.08$, $\min =-0.12$, $\max =-0.02$ ) and low performance for the simpler model (median $\mathrm{R} 2=0.01, \min =-0.01, \max =0.03$ ).

Our main goal was to investigate which were the primary factors that modulated time awareness. To interpret the model's decisions, we used SHapley Additive exPlanations - SHAP (Scott M. Lundberg et al., 2020; S. Lundberg \& Lee, 2017) which allows us to understand the role of different features in the model's decisions. The SHAP value shows the average contribution of a feature value to the model's prediction (Molnar, 2019). Critically, SHAP values show which features the algorithm is using to make its predictions, but not the actual relationship between that feature of interest and the dependent variable.

Figure 4A shows, for both measures of time awareness, the top 25\% of the features (top 12 features out of 47) that had the highest SHAP values, while Figures 4B and Figures 4C show, in more detail, the SHAP values for the top $10 \%$ (5 features out of 47 ). To illustrate further the association between features and the dependent variables, the lower panels of Figures 4B and 4C show the observed time expansion and time pressure scores as a function of the features with the highest SHAP values.

To understand Figure 4 and the relationship between SHAP values, feature values and the dependent variable (i.e., time expansion or time pressure), consider the following example: Take the role of loneliness in modulating the model's output about time expansion. In the SHAP summary plot (Figure 4A), note that higher values of loneliness (darker shades of green) 
are associated with higher SHAP values (x-axis). The origin point on the $\mathrm{x}$-axis represents the model's output if all features were unknown. As can be seen, high values of loneliness contribute to adding up to 10 points to the predicted score of time expansion. The relationship between loneliness and the SHAP values for this feature can be explored further in Figure 4B, suggesting that this relation is not entirely linear. As previously mentioned, SHAP values show how each feature contributes to the model's predictions, not the actual relationship between a given feature and the response variable (in this example, time expansion). The lower panels from Figure 4B (and Figure 4C for time pressure) show each of these features binned in five equally spaced intervals with the observed time awareness scores to illustrate the relationship between the most relevant features of the model with the actual response data. As Figure 4B suggests, the relationship pattern between the SHAP values and actual values of loneliness closely follow the association between loneliness and time expansion, and that the later indicates that increasing levels of loneliness are associated with increased perception of time slowing down.

For time expansion, the features that had a stronger influence on the models' predictions about reported increases/decreases were: (1) Time expansion before social isolation, with higher reports of temporal expansion pre-social isolation being associated with predictions of higher reports of temporal expansion during social isolation; (2) Feeling of loneliness, with stronger feelings of loneliness being related to predictions of higher reports of time expansion; (3) Positive affect (as measured by PANAS), with lower scores of positive emotions being associated with predictions of higher reports of time expansion; (4) Age, being younger participants related to predictions of reporting higher values of time expansion; (5) Negative affect (as measured by PANAS), being higher scores of negative emotions associated with predictions of higher reports of time expansion.

For time pressure, the features that had a stronger influence on the models' predictions about reported increases/decreases were: (1) Personal care, being lower values of personal care associated with predictions of higher reports of temporal pressure during social isolation; (2) Time pressure in the period before social isolation, with higher reports of time pressure pre-social isolation being associated predictions of with higher reports of time pressure during 
social isolation; (3) Perceived stress (as measured by PSS-10), being higher stress levels associated with predictions of higher reports of time pressure; (4) Hours spent working and studying, being a higher number of hours related to predictions of higher values of time pressure; (5) Positive affect (as measured by PANAS), being higher scores of positive emotions associated with predictions of higher reports of time pressure.

Overall, results for the first week of the survey show that participants' time awareness changed after social isolation measures were implemented. This was true both for feelings of time being empty (i.e., time expansion) and feelings of time running out (i.e., time pressure). Time awareness in the first session was best predicted by time awareness before social isolation, affect, stress, feeling of loneliness, and some everyday life activities (self-care and working hours); age was the only relevant demographic. 
A
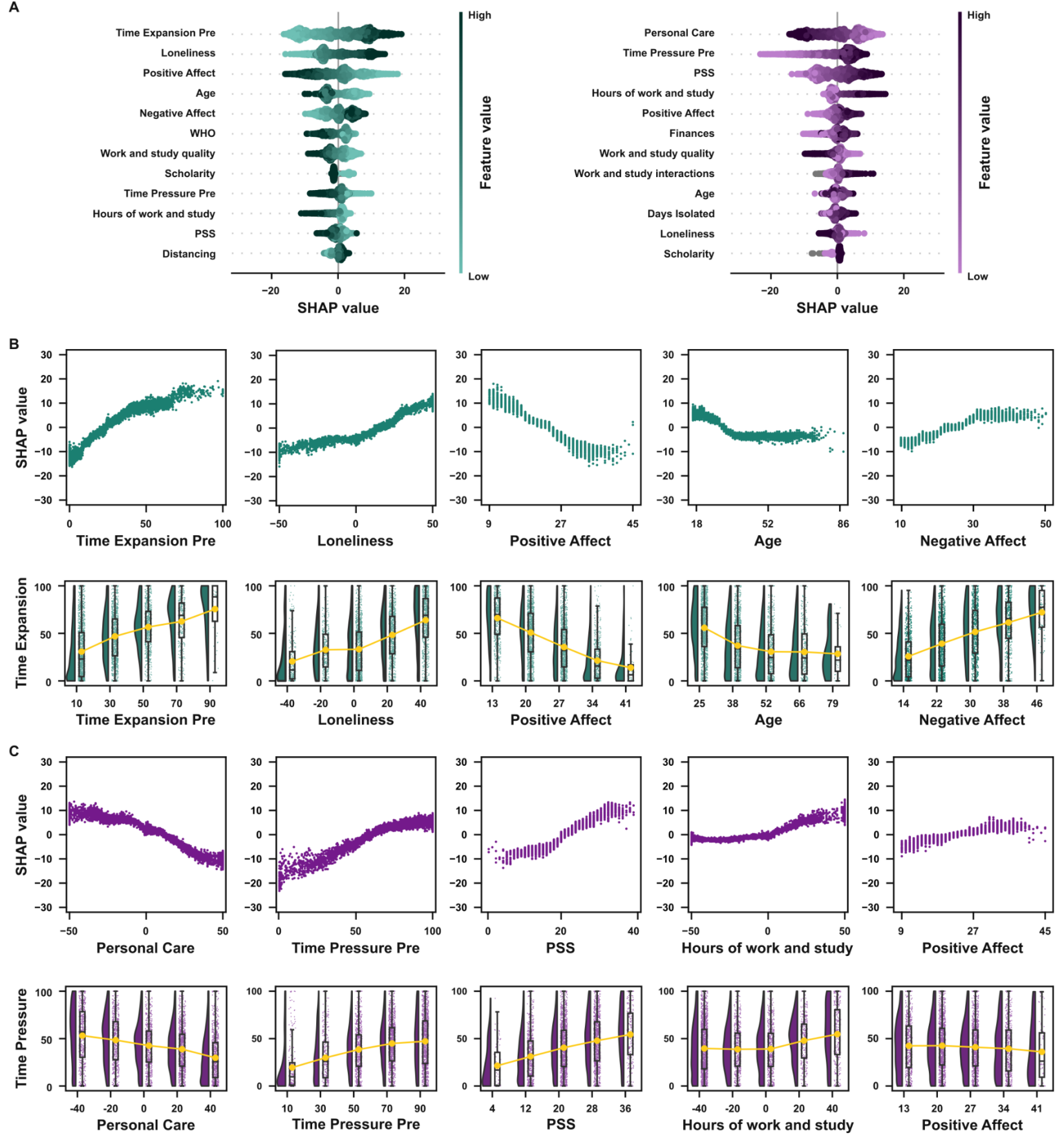

Figure 4. Factors associated with distortions in the time awareness in the first session (A) SHAP summary plots for time expansion (left panel) and time pressure (right panel). The summary plots show a combination of feature importance with feature effects. Each point is a SHAP value for a feature and an instance. Each line represents a feature, and the x-axis is the SHAP value (on the same scale as the time-awareness VAS). The color represents the value of the feature from low to high. Overlapping points are jittered in the $y$-axis direction, allowing a sense of the distribution of the SHAP values per feature. Features are sorted according to their importance. (B) Factors associated with distortions in time expansion. Upper panel show SHAP dependence plots for the five features with the highest SHAP values for time expansion. Each plot shows, for each data instance, the feature value on the $x$-axis and the corresponding SHAP value on the y-axis. The SHAP value describes the behavior of the model for different values of a given feature. For example, a low score on positive affect predicted a decrease in the average value of time expansion. In contrast, high scores on positive affect predicted higher scores of time expansion. Lower panels show raincloud plots for each feature. The feature of interest is binned in five equally distanced values in each plot, and time expansion values for each bin are shown. (C) Factors associated with distortions in time pressure. The plots follow the same patterns as (B). 


\section{Evolution of time expansion and pressure during social isolation}

As the weeks of social isolation continued, we investigated whether participants' time awareness changed over time. As a starting point to understand such fluctuations in time awareness, we estimated participants' median absolute deviation (MAD) over weeks for time expansion and pressure as a coarse measure of response variability. Participants' responses varied across the weeks [time expansion: median-MAD $(\mathrm{IQR})=6.485(3.179,10.359)$; time pressure: median-MAD $(I Q R)=6.273(3.149,10.275)]$, suggesting changes in how they perceive time across weeks of social isolation.

We next investigated whether there was a monotonic increase/decrease of time pressure and expansion throughout the weeks. For each participant, we calculated the Spearman correlation coefficient (rho) between time awareness scores and week and, at the group level, compared the estimated rhos against zero using a sign-test (Figure 5). There was a small decrease of time expansion throughout the weeks [median rho $(\mathrm{IQR})=-0.086(-0.5,0.369), \mathrm{p}=$ 0.001 , Cohen's $\left.g=-0.054^{2}\right]$, and no significant association between week and time pressure [median rho $(\mathrm{IQR})=0.051(-0.4,0.5), \mathrm{p}=0.078$, Cohen's $g=0.029]$. Together, these results suggest that, although there were weekly variations on time awareness, these variations were not well explained by a simple increase/decrease as a function of the week.

Factors associated with distortions in the time awareness during weekly sessions

We performed a similar characterization of the magnitude of weekly fluctuations and possible weekly monotonic increase/decrease for all scales that participants filled in their weekly sessions. As can be seen in Table 3, the groups of questions that more consistently increase/decrease during the weeks were related to changes in routine, opinions on social distancing, and quality of life. Once again, it is important to emphasize that the MAD analysis is used just to verify whether there were fluctuations in participants' responses across the

${ }^{2}$ Cohen's $g$ is used as a measure of effect size for sign tests in conditions where the expected proportion in the population is 0.5 (50\%). It is calculated as the simple difference of the sample proportion in relation to the expected proportion. Positive values indicate that the observed proportion was above 0.5 and negative values indicate that it was below 0.5 (Rosnow, 2003). 
weeks, but only the Spearman correlation analysis can inform whether there was a monotonic increase/decrease over weeks.
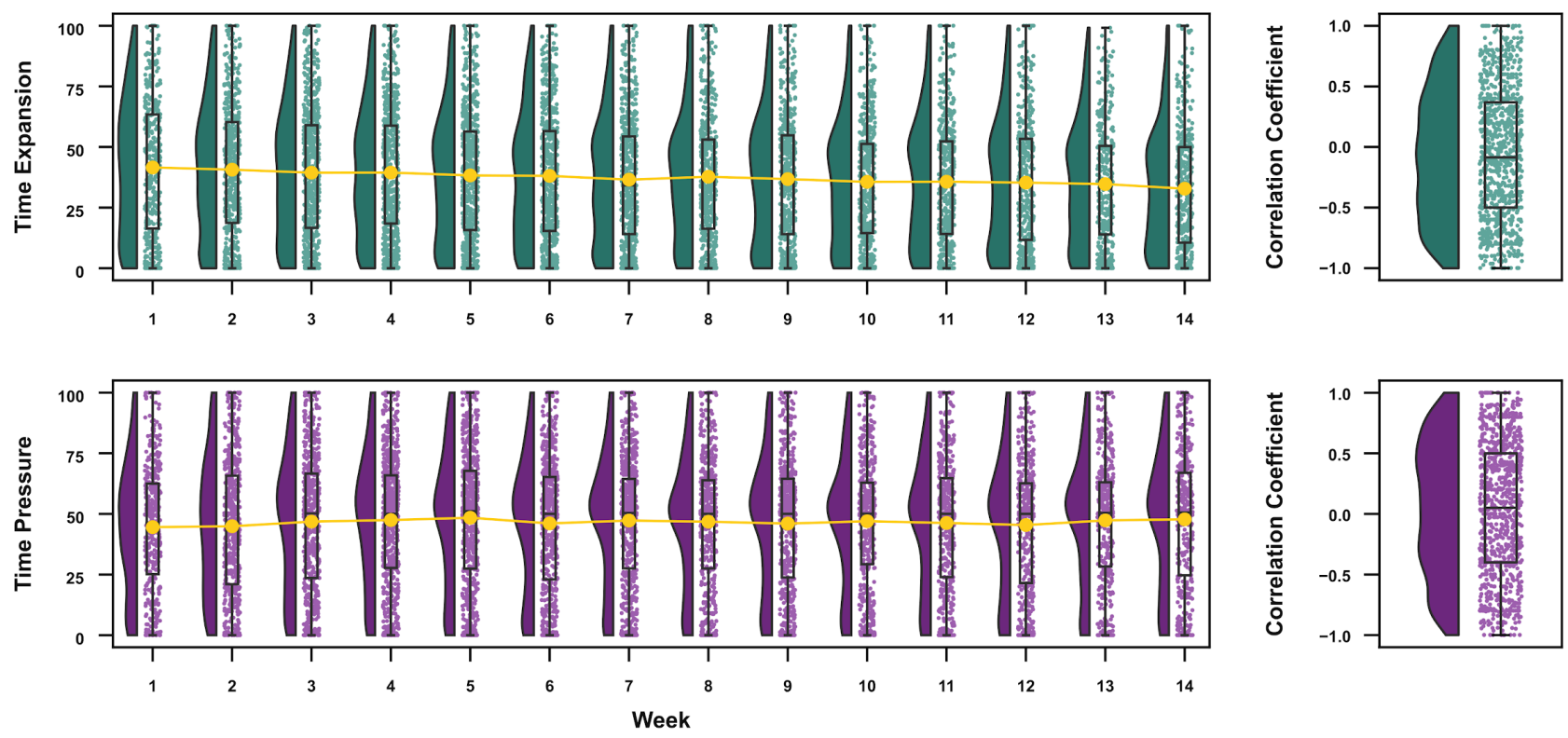

Figure 5. Evolution of time awareness during social isolation. Raincloud plots showing group distributions as un-mirrored violin plots (probability density functions), boxplots with medians and interquartile ranges, whiskers with 5 th and 95th percentiles, and individual data points (horizontally jittered). Panels on the right show the distribution of correlation coefficients between week and time expansion and pressure across participants. 


\begin{tabular}{|c|c|c|c|c|c|c|}
\hline Feature & MAD & IQR-MAD & Median Rho & IQR & $\begin{array}{l}\text { Effect size } \\
\text { (Cohen's g) }\end{array}$ & p-value \\
\hline \multicolumn{7}{|c|}{ Affect (PANAS Scale) } \\
\hline Positive Affect & 2.196 & {$[1.0,3.0]$} & -0.036 & {$[-0.463,0.359]$} & -0.024 & 0.604 \\
\hline Negative Affect & 2.334 & {$[1.0,3.0]$} & -0.286 & {$[-0.6,0.158]$} & -0.179 & $<0.001$ \\
\hline \multicolumn{7}{|c|}{ Stress and Well-Being } \\
\hline PSS & 2.027 & {$[1.0,3.0]$} & -0.109 & {$[-0.527,0.257]$} & -0.069 & $<0.001$ \\
\hline WHO & 1.496 & {$[1.0,2.0]$} & 0.062 & {$[-0.364,0.463]$} & 0.048 & 0.032 \\
\hline \multicolumn{7}{|c|}{ Sense of Isolation } \\
\hline Loneliness & 7.634 & {$[1.0,11.5]$} & -0.046 & {$[-0.4,0.371]$} & -0.027 & 0.539 \\
\hline Distancing & 8.242 & {$[2.0,12.0]$} & -0.188 & {$[-0.518,0.258]$} & -0.113 & $<0.001$ \\
\hline Personal Interactions & 7.43 & {$[1.5,11.0]$} & 0.036 & {$[-0.336,0.4]$} & 0.03 & 0.51 \\
\hline $\begin{array}{l}\text { Work and study } \\
\text { interactions }\end{array}$ & 7.003 & {$[1.0,10.5]$} & 0.029 & {$[-0.4,0.4]$} & 0.024 & 0.656 \\
\hline Personal Care & 8.336 & {$[2.0,12.0]$} & 0 & {$[-0.4,0.359]$} & -0.004 & 0.818 \\
\hline \multicolumn{7}{|c|}{ Opinion on social distancing } \\
\hline Opinion on helping & 5.711 & {$[1.0,8.5]$} & -0.348 & {$[-0.677,0.137]$} & -0.197 & $<0.001$ \\
\hline Agreement & 8.198 & {$[1.0,12.0]$} & -0.4 & {$[-0.725,0.0]$} & -0.25 & $<0.001$ \\
\hline News & 7.396 & {$[2.0,11.0]$} & -0.4 & {$[-0.699,0.056]$} & -0.223 & $<0.001$ \\
\hline Self Risk & 6.614 & {$[2.0,9.5]$} & 0.167 & {$[-0.285,0.535]$} & 0.106 & $<0.001$ \\
\hline Others' Risk & 5.476 & {$[1.0,8.0]$} & 0.047 & {$[-0.377,0.426]$} & 0.029 & 0.539 \\
\hline \multicolumn{7}{|c|}{ Quality of Life } \\
\hline Finances & 4.256 & {$[0.0,7.0]$} & 0.027 & {$[-0.396,0.429]$} & 0.019 & 0.656 \\
\hline Work and study quality & 6.197 & {$[1.0,9.0]$} & 0.094 & {$[-0.321,0.441]$} & 0.058 & 0.005 \\
\hline Leisure & 6.168 & {$[1.0,9.5]$} & 0.11 & {$[-0.348,0.516]$} & 0.066 & 0.001 \\
\hline Family & 6.23 & {$[1.0,10.0]$} & 0.067 & {$[-0.336,0.467]$} & 0.052 & 0.019 \\
\hline Exercises & 6.414 & {$[0.5,10.0]$} & 0.082 & {$[-0.331,0.476]$} & 0.045 & 0.056 \\
\hline \multicolumn{7}{|c|}{ Routine changes } \\
\hline $\begin{array}{l}\text { Hours of work and } \\
\text { study }\end{array}$ & 6.15 & {$[0.0,9.0]$} & 0.082 & {$[-0.318,0.447]$} & 0.058 & 0.007 \\
\hline Hours of leisure & 3.463 & {$[0.0,4.5]$} & 0.3 & {$[-0.101,0.65]$} & 0.192 & $<0.001$ \\
\hline $\begin{array}{c}\text { Interaction with } \\
\text { housemates }\end{array}$ & 5.658 & {$[0.0,8.5]$} & -0.143 & {$[-0.498,0.219]$} & -0.098 & $<0.001$ \\
\hline Hours spent online & 7.321 & {$[2.0,11.0]$} & -0.182 & {$[-0.516,0.224]$} & -0.11 & $<0.001$ \\
\hline Hours spent outside & 3.829 & {$[0.0,4.5]$} & 0.258 & {$[-0.188,0.61]$} & 0.166 & $<0.001$ \\
\hline Relaxation & 7.315 & {$[2.75,11.75]$} & 0.296 & {$[-0.289,0.701]$} & 0.155 & $<0.001$ \\
\hline
\end{tabular}


Table 3. Evolution of the different scales during social isolation. Description of the groups of questions that more consistently increased/decreased during the weeks. The first two columns (MAD and IQR-MAD) show the variation of that measure across weeks. Median Rho and IQR shows the median Rho between that measure and week across participants. We used a binomial sign test across participants to test whether there was consistent increase/decrease of that measure over the weeks. Cohen's g shows the effect size (proportion of participants with a positive Rho - 0.5 ) and the respective p-value, corrected for multiple comparisons using Holm's method.

To investigate whether different measures covaried across weeks, we calculated pairwise correlations of all scales that were completed weekly and the median correlation across participants. To illustrate further the associations between different measures, we used hierarchical cluster analysis to build a dendrogram in which scales that are more strongly associated are placed on branches closer together (Figure 6). Once again, weekly time expansion reports were more associated with well-being and loneliness, while time pressure was more correlated with stress and personal care. 

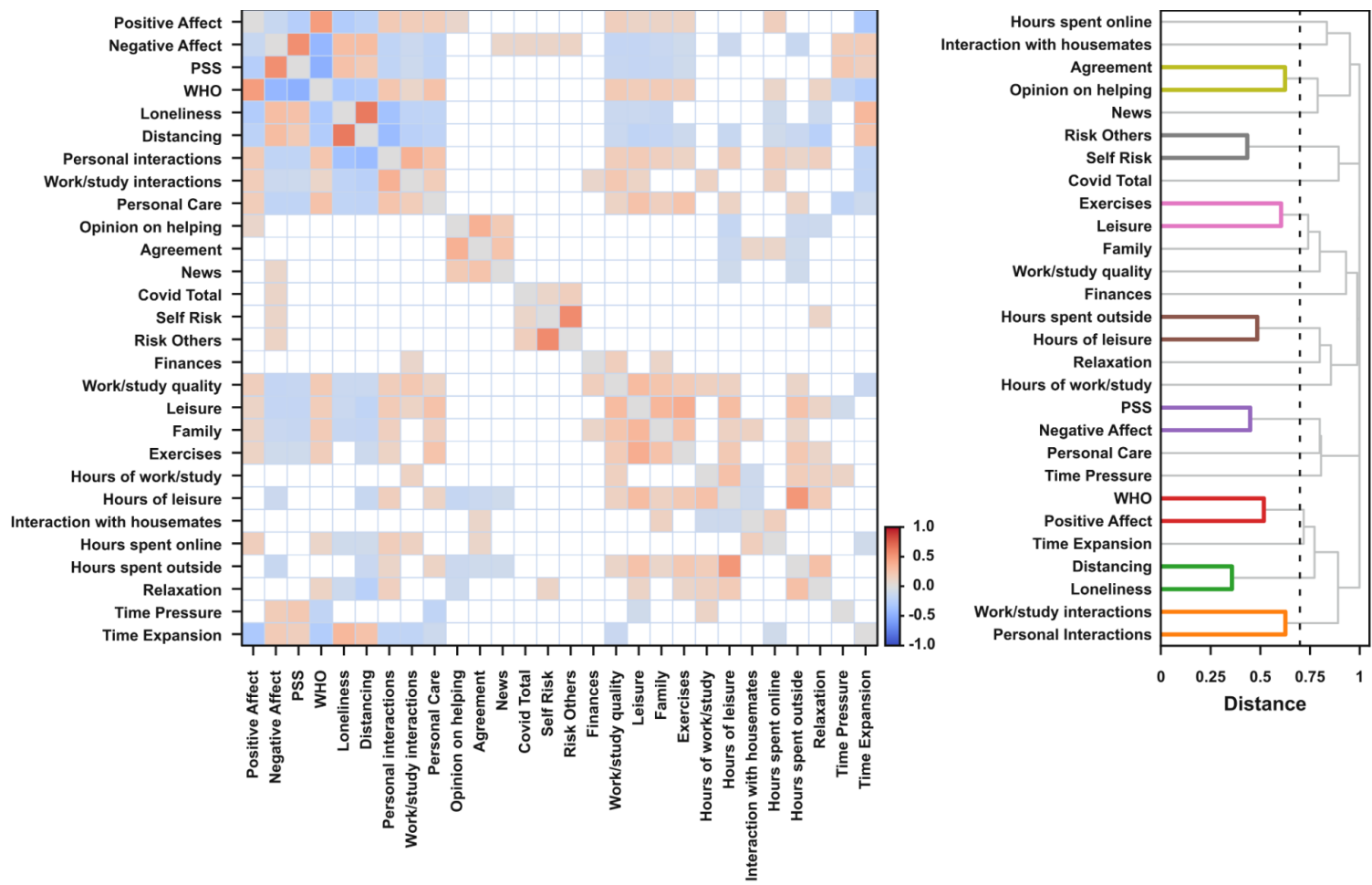

Figure 6. Pairwise correlations between measures and hierarchical cluster analysis during the weekly sessions. Left panel shows the pairwise Spearman correlations of across measures from weekly sessions. Only effects stronger than 0.1 are represented. The colour of individual points indicates the sign and strength of estimated coefficients. Right panel shows the estimated dendrograms using hierarchical clustering analyses of the estimated distances between scales (Distance $=1-\mid$ rho| $)$. The black vertical lines represent the cut threshold, which was defined at a distance between scales with a correlation of medium effect size $(\mid$ rhol $=0.3)$. Links connecting nodes with distances smaller than or equal to the threshold are marked as belonging to the same cluster.

We performed a similar gradient boosting regression to examine which factors were associated with stronger feelings of time expansion and pressure during the weekly sessions. Data from all weekly sessions were collapsed and a single model was fit. For time expansion, once again, the full model had good predictive scores (median $\mathrm{R} 2=0.43, \min =0.32, \max =$ 0.52), compared to the chance levels for the shuffled model (median $\mathrm{R} 2=-0.06$, $\min =-0.15$, $\max =0.03)$ and low performance for the simpler model (median $\mathrm{R} 2=0.04, \min =-0.17, \max =$ 0.17). A similar pattern was found for time pressure, with good performance for the full model $($ median $\mathrm{R} 2=0.27, \min =0.12, \max =0.38)$, chance levels for the shuffled model $($ median $\mathrm{R} 2=$ 
$-0.06, \min =-0.14, \max =0.02)$ and low performance for the simpler model (median $\mathrm{R} 2=-0.01$, $\min =-0.22, \max =0.12$ ).

Figure 7 shows, for both measures of time awareness, the top $25 \%$ of the features (A) and the relationship in the top $10 \%$ (B and C) features that had the highest SHAP values. For time expansion, the features that had a stronger influence on the models' predictions on reported increases/decreases were: (1) Positive affect (as measured by PANAS), with lower scores of positive emotions being associated with predictions of higher reports of time expansion; (2) Time expansion in the period before social isolation, with higher reports of temporal expansion pre-social isolation being associated with predictions of higher reports of temporal expansion during social isolation; (3) Feeling of loneliness, with stronger feelings of loneliness being associated with predictions of higher reports of time expansion; (4) Well-being, (as measured by WHO-5), with lower values being associated with predictions of higher reports of time expansion; (5) Time pressure in the period before social isolation, with higher reports of time pressure pre-social isolation being associated with predictions of lower reports of time expansion during social isolation.

For time pressure, the features that had a stronger influence on the models' predictions on reported increases/decreases were: (1) Perceived stress (as measured by PSS-10), with higher stress levels being associated with predictions of higher reports of time pressure; (2) Time pressure in the period before social isolation, with higher reports of time pressure pre-social isolation being associated with predictions of higher reports of time pressure during social isolation; (3) Personal care, with lower values of personal care being associated with predictions of higher reports of temporal pressure; (4) Work and study interactions, with more interactions being associated with predictions of higher values of time pressure; (5) Work and study quality, with reported worse quality hours being associated with predictions of higher values of time pressure.

Overall, results from the first session and weekly sessions converge towards the same conclusion: time awareness was distorted during social isolation. Time expansion was strongly associated with emotions and feelings of isolation, and time pressure was strongly associated with perceived stress and personal care. 
A

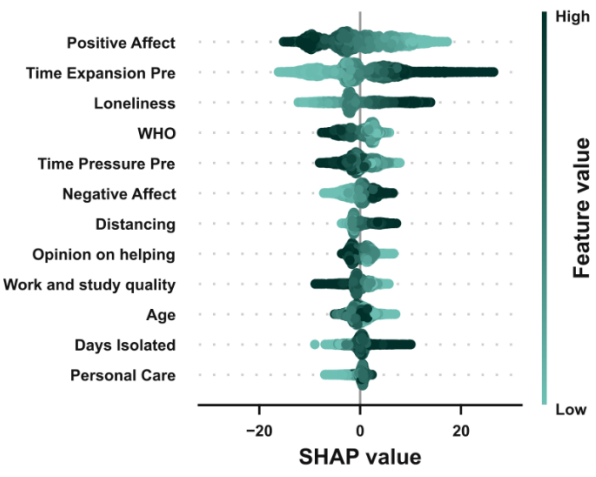

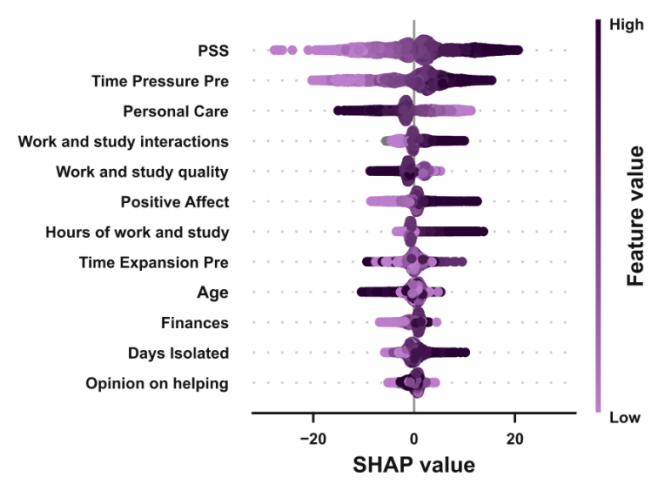

B
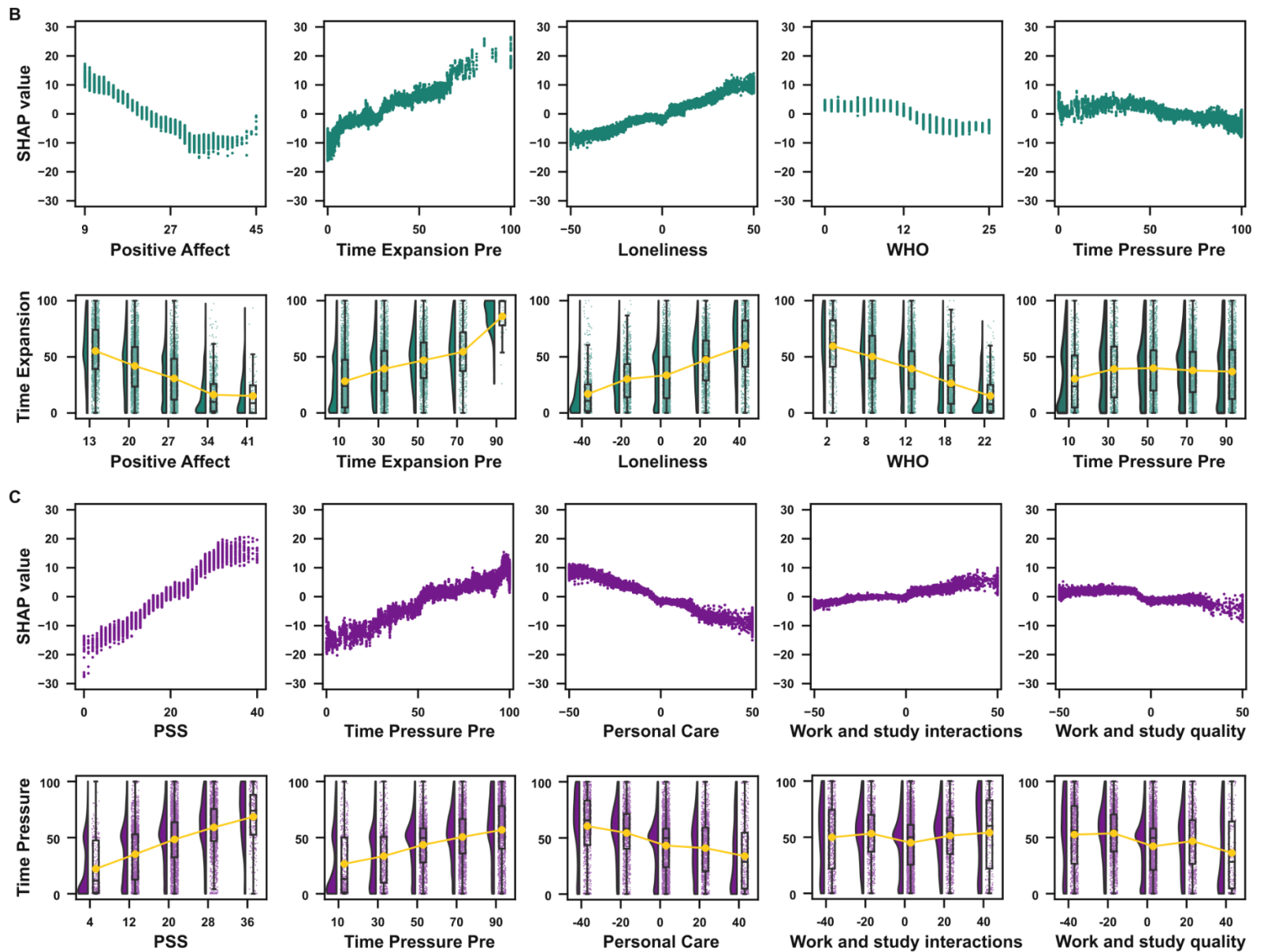

Figure 7. Factors associated with distortions in the time awareness during the weekly sessions, (A) SHAP summary plots for time expansion (left panel) and time pressure (right panel). The summary plots show a combination of feature importance with feature effects. Each point is a SHAP value for a feature and an instance. Each line represents a feature, and the x-axis is the SHAP value (on the same scale as the time-awareness VAS). The color represents the value of the feature from low to high. Overlapping points are jittered in the $y$-axis direction, allowing a sense of the distribution of the SHAP values per feature. Features are ordered according to their importance. (B) Factors associated with distortions in time expansion. Upper panel show SHAP dependence plots for the five features with the highest SHAP values for time expansion. Each plot shows, for each data instance, the feature value on the x-axis and the corresponding SHAP value on the $y$-axis. The SHAP value describes the behavior of the model for different values of a given feature. Lower panels show raincloud plots for each feature. The feature of interest is binned in five equally distanced values in each plot, and time expansion values for each bin are shown. (C) Factors associated with distortions in time pressure. The plots follow the same patterns as (B). 


\section{Temporal estimates}

Before completing the questionnaires, participants had to produce temporal intervals of 1, 3 and 12 s (three times each). Participants were prompted with a screen where they had to press a button to start the interval and press it again when they thought the corresponding time interval to be produced had ended. As shown in Figure 8, participants were able to correctly perform the task as evidenced by the median produced intervals being close to the target time interval and clear differences across target intervals [Friedman test: $Q(2)=6966.61, p<0.001$; Paired Wilcoxon post-hoc: $\mathrm{W}_{3-12}=1532, \mathrm{~W}_{3-1}=75449, \mathrm{~W}_{12-1}=5$, all $\left.\mathrm{p}<0.001\right]$. Estimation accuracy differed depending on the target interval: one second intervals were overestimated [median $(\mathrm{IQR})=1.32(1-1.72), \mathrm{p}-\mathrm{Holm}<0.001$, Cohen's $\mathrm{g}=0.253$ ], three second intervals were not significantly different than 3 seconds [median $(\mathrm{IQR})=3.04(2.37-3.8), \mathrm{p}-\mathrm{Holm}=0.086$, Cohen's $g=0.014$ ], while twelve second intervals were underestimated [median $(\mathrm{IQR})=10.91$ (8.4-13.21), -Holm < 0.001, Cohen's g $=-0.123]$.

One of our goals was to check whether time awareness was related to interval timing and whether the difference in time awareness due to social isolation affected participants' internal clocks. To measure these possible relations we performed an across participants Shepherd correlation (a robust version of Spearman correlation, (Rousselet \& Pernet, 2012) between the produced interval (averaged separately for 1, 3 and 12s) and the time awareness scales. We included four scales in our analysis: (1) time pressure during social isolation; (2) time expansion during social isolation; (3) the difference between time pressure during social isolation and before social isolation; (4) the difference between time expansion during social isolation and before social isolation. These two last scales were included to investigate whether participants who had larger distortions of time awareness caused by social isolation also had a higher distortion in temporal production. However, for all comparisons, the estimated correlations were weak and not significant $[$ rho $=(-0.028,0.038)$, all Holm corrected $\mathrm{p}$-values $>$ 0.08]. 


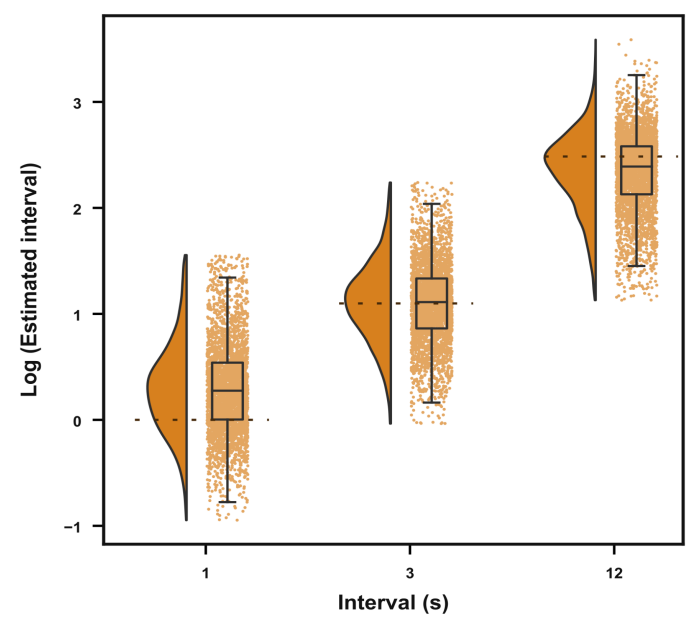

Figure 8. Produced intervals in the first session. Produced intervals (log-transformed) for 1,3 and 12s. Raincloud plots showing group distributions as un-mirrored violin plots (probability density functions), boxplots with means and interquartile ranges, whiskers with 5th and 95th percentiles, and individual data points - horizontally jittered (adapted from (Allen et al., 2019)).

\section{Evolution of temporal estimates during social isolation}

We were interested in investigating not only if time awareness was distorted during social isolation but also whether the production of intervals on the seconds-range was affected across the weeks. Figure 9 shows the distribution of the temporal productions across the weeks. In general, participants produced shorter intervals along the weeks irrespective of the interval to be estimated [1s rho median $(\mathrm{IQR})=-0.12(-0.48,0.29)$, $\mathrm{p}-\mathrm{Holm}<0.001$, Cohen's $\mathrm{g}=-0.075$; $3 \mathrm{~s}$ rho median $(\mathrm{IQR})=-0.12(-0.5,0.28), \mathrm{p}-\mathrm{Holm}<0.001$, Cohen's $\mathrm{g}=-0.092 ; 12 \mathrm{~s}$ rho median $(\mathrm{IQR})=-0.1(-0.49,0.29), \mathrm{p}-\mathrm{Holm}<0.001$, Cohen's $\mathrm{g}=-0.065]$.

To understand whether the decrease of the produced intervals across weeks was related to time awareness, we conducted, for each participant, Spearman partial correlations between interval productions and time awareness scores controlling for the effect of week. The produced intervals were not correlated with time awareness (Figure 9B), neither with time expansion [1s rho median $(\mathrm{IQR})=0(-0.38,0.35), \mathrm{p}-\mathrm{Holm}=1$, Cohen's $\mathrm{g}=0$; 3s rho median $(\mathrm{IQR})=-0.03$ $(-0.36,0.37), \mathrm{p}-\mathrm{Holm}=0.485$, Cohen's $\mathrm{g}=-0.024 ; 12 \mathrm{~s}$ rho median $(\mathrm{IQR})=0(-0.38,0.36)$, $\mathrm{p}$-Holm $=1$, Cohen's $\mathrm{g}=-0.008]$; nor with time pressure $[1 \mathrm{~s}$ rho median $(\mathrm{IQR})=-0.03(-0.39$, $0.31), \mathrm{p}-$ Holm $=0.18$, Cohen's $\mathrm{g}=-0.029 ; 3 \mathrm{~s}$ rho median $(\mathrm{IQR})=-0.03(-0.4,0.33)$, $\mathrm{p}-\mathrm{Holm}=$ 
0.18, Cohen's $g=-0.023 ; 12 \mathrm{~s}$ rho median $(\mathrm{IQR})=-0.04(-0.4,0.3)$, $\mathrm{p}-\mathrm{Holm}=0.084$, Cohen's $\mathrm{g}=$ $-0.037]$.

In addition, the decrease of the produced intervals across weeks was still present when controlling for the correlations with time expansion and pressure [Figure 9B; partial Spearman correlations: 1s rho median $(\mathrm{IQR})=-0.1(-0.55,0.45), \mathrm{p}-\mathrm{Holm}=0.003$, Cohen's $\mathrm{g}=-0.052 ; 3 \mathrm{~s}$ rho median $(\mathrm{IQR})=-0.11(-0.6,0.4), \mathrm{p}-\mathrm{Holm}<0.001$, Cohen's $\mathrm{g}=-0.063 ; 12 \mathrm{~s}$ rho median $(\mathrm{IQR})=$ $-0.1(-0.6,0.41), \mathrm{p}-\mathrm{Holm}=0.004$, Cohen's $\mathrm{g}=-0.048]$. Overall, these results indicate that the decrease of the produced intervals across weeks cannot be explained by time awareness and social isolation. 
A
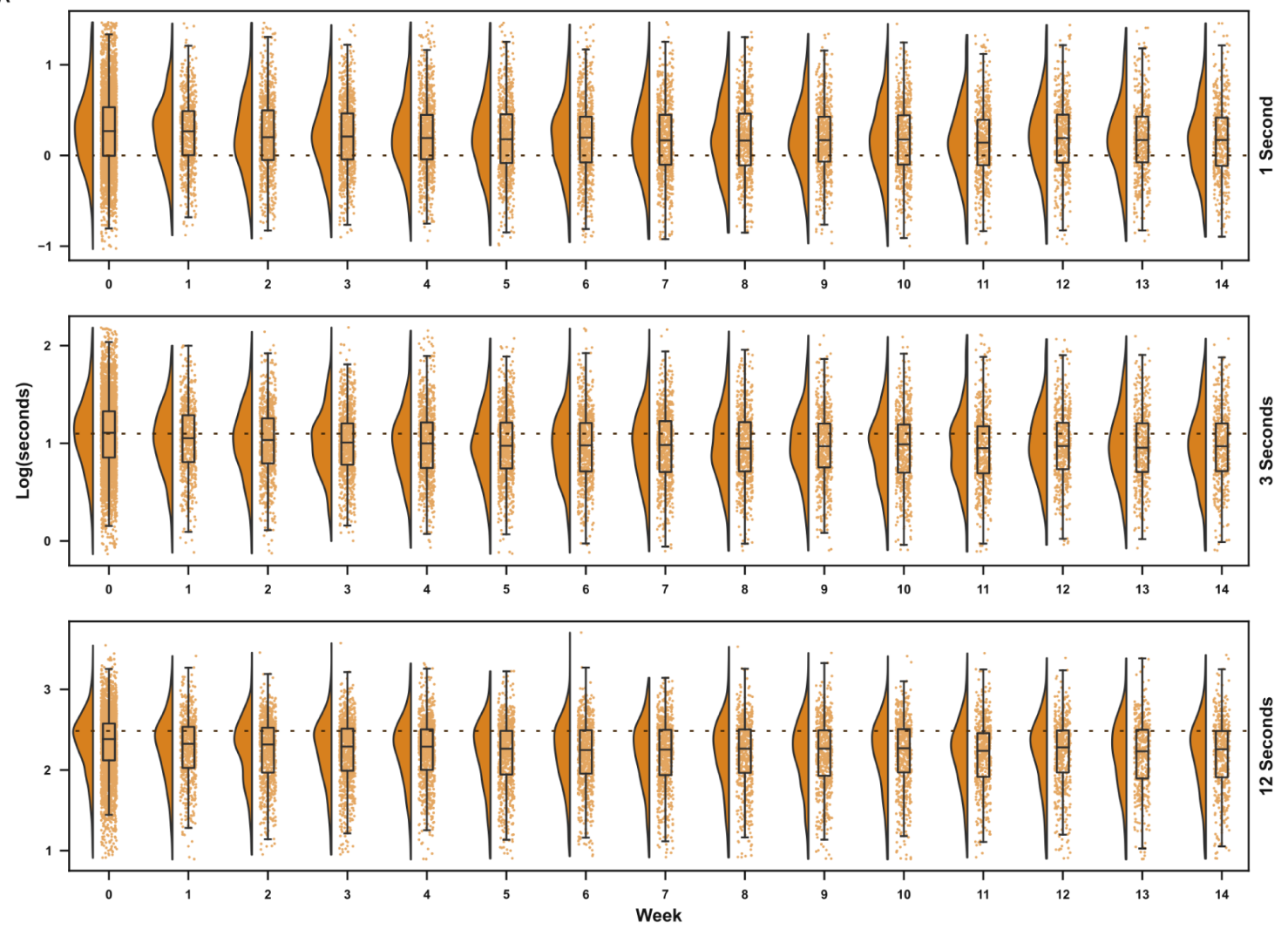

B
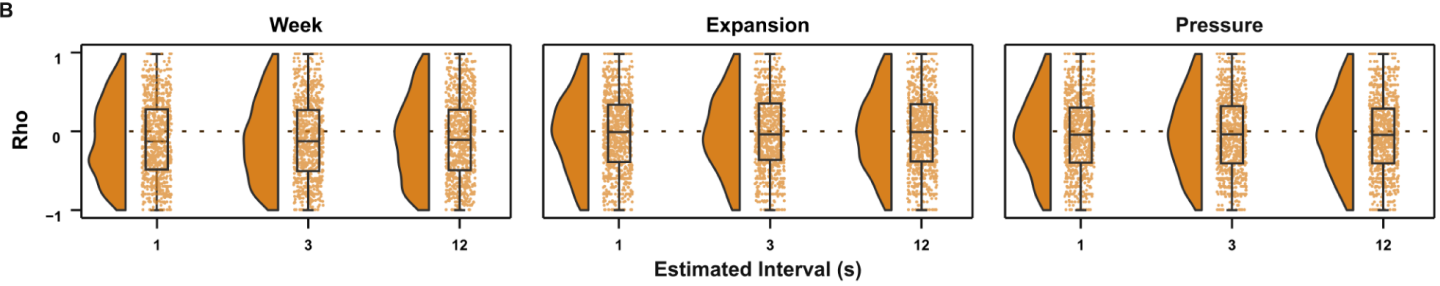

Figure 9. Evolution of temporal productions across weeks. (A) Distribution of participants' estimates for 1,3 and $12 \mathrm{~s}$ time intervals (log-transformed) across the weeks. (B) Coefficients' distribution from within-participant partial Spearman correlations between temporal estimates and i) week, considering time expansion and time pressure as covariates; ii) time expansion with week as a covariate; iii) time pressure with week as a covariate. 


\section{Natural Language Processing of Open Questions}

\section{$\underline{\text { First Session }}$}

At the end of the survey, participants were invited to complete two open questions: (1) To briefly share the external facts (news, events) that had marked them the most since the beginning of the social isolation; (2) To report what had personally marked them the most since the beginning of the social isolation. We explored whether we could extract the emotional content of the responses using Natural Language Processing (NLP) tools and whether the extracted emotional contents were correlated with time awareness. To extract the emotional context from these open-ended questions, responses were analyzed using the Stanford Sentiment Treebank (Socher et al., 2013), and classified as reports with a very negative, negative, neutral, positive or very positive polarity.

As can be seen in Table 4, we found that the polarity of personal reports was correlated with stress (as measured by PSS-10), well-being (as measured by WHO-5), positive and negative emotions (as measured by PANAS) and time expansion during the period of social isolation. The polarity of external news and facts were correlated with negative emotions, stress, well-being, loneliness, exercises and time pressure during the period of social isolation. 


\begin{tabular}{|c|c|c|c|c|}
\hline & \multicolumn{2}{|c|}{ Personal reports } & \multicolumn{2}{|c|}{ News and external facts } \\
\hline Feature & rho & p-value & rho & p-value \\
\hline \multicolumn{5}{|c|}{ Time Awareness } \\
\hline Time expansion during social isolation & -0.089 & $<0.001$ & -0.058 & 0.072 \\
\hline Time pressure during social isolation & -0.027 & 1.000 & -0.075 & 0.004 \\
\hline Time expansion before social isolation & -0.018 & 1.000 & -0.039 & 0.814 \\
\hline Time pressure before social isolation & -0.025 & 1.000 & 0.009 & 1.000 \\
\hline \multicolumn{5}{|c|}{ Affect (PANAS Scale) } \\
\hline Positive Affect & 0.103 & $<0.001$ & 0.042 & 0.63 \\
\hline Negative Affect & -0.124 & $<0.001$ & -0.065 & 0.03 \\
\hline \multicolumn{5}{|c|}{ Stress and Well-Being } \\
\hline PSS & -0.109 & $<0.001$ & -0.072 & 0.01 \\
\hline WHO & 0.108 & $<0.001$ & 0.086 & $<0.001$ \\
\hline \multicolumn{5}{|c|}{ Sense of Isolation } \\
\hline Loneliness & -0.053 & 0.179 & -0.077 & $<0.001$ \\
\hline Distancing & -0.055 & 0.140 & -0.010 & 1.00 \\
\hline Personal Interactions & 0.008 & 1.000 & -0.003 & 1.00 \\
\hline Work and study interactions & 0.023 & 1.000 & 0.025 & 1.00 \\
\hline Personal Care & 0.014 & 1.000 & 0.038 & 0.81 \\
\hline \multicolumn{5}{|c|}{ Opinion on social distancing } \\
\hline Opinion on helping & 0.023 & 1.000 & -0.012 & 1.00 \\
\hline Agreement & -0.004 & 1.000 & -0.030 & 1.00 \\
\hline News & -0.007 & 1.000 & -0.001 & 1.00 \\
\hline Self Risk & 0.005 & 1.000 & -0.047 & 0.37 \\
\hline Others' Risk & -0.024 & 1.000 & -0.009 & 1.00 \\
\hline \multicolumn{5}{|c|}{ Quality of Life } \\
\hline Finances & -0.002 & 1.000 & 0.012 & 1.00 \\
\hline Work and study quality & 0.051 & 0.225 & 0.002 & 1.00 \\
\hline Leisure & -0.017 & 1.000 & 0.033 & 1.00 \\
\hline Family & -0.003 & 1.000 & 0.060 & 0.06 \\
\hline Exercises & 0.014 & 1.000 & 0.065 & 0.03 \\
\hline \multicolumn{5}{|c|}{ Routine changes } \\
\hline Hours of work and study & -0.023 & 1.000 & 0.016 & 1.00 \\
\hline Hours of leisure & -0.012 & 1.000 & 0.023 & 1.00 \\
\hline Interaction with housemates & 0.045 & 0.481 & 0.042 & 0.63 \\
\hline Hours spent online & 0.016 & 1.000 & 0.045 & 0.45 \\
\hline Hours spent outside & 0.003 & 1.000 & 0.039 & 0.81 \\
\hline
\end{tabular}


Table 4. Correlation between the emotional valence of reports and measures scales. A Shepherd's correlation between the estimated valence of the reports across participants. The $p$-values were corrected for multiple comparisons using Holm's method.

\section{Evolution of reports' polarity during social isolation}

We investigated whether, within participants, the polarity of the reported news and personal reports were correlated with week, time awareness and the other scales. As can be seen in Table 5, the polarity of personal reports was correlated with positive emotions and well-being. The reported news, on the other hand, did not significantly correlate with other measures.

Taken together, the NLP results showed that in the first session, the polarity of personal reports was correlated with time expansion while the polarity of the news and external facts correlated with time pressure. This result agrees with the general finding of time expansion being more related to personal and psychological factors. In contrast, time pressure is more associated with a mixture of psychological and stress-related factors. We did not observe correlations between time awareness and the polarity of either personal reports or the external facts participants shared for the weekly sessions. Nevertheless, the polarity of personal reports was still correlated with general well-being measures, showing the potential power of this kind of analysis for future studies. 


\begin{tabular}{|c|c|c|c|c|c|c|c|c|}
\hline & \multicolumn{4}{|c|}{ Personal reports } & \multicolumn{4}{|c|}{ News and external facts } \\
\hline Feature & $\begin{array}{l}\text { Median } \\
\text { Rho }\end{array}$ & IQR & $\begin{array}{l}\text { Effect size } \\
\text { (Cohen's g) }\end{array}$ & p-value & $\begin{array}{l}\text { Median } \\
\text { Rho }\end{array}$ & IQR & $\begin{array}{l}\text { Effect size } \\
\text { (Cohen's g) }\end{array}$ & $p$-value \\
\hline \multicolumn{9}{|c|}{ Week } \\
\hline Week & 0.039 & $\begin{array}{c}-0.307 \\
0.359]\end{array}$ & 0.035 & 1 & 0.073 & $\begin{array}{c}-0.259 \\
0.36]\end{array}$ & 0.075 & 0.056 \\
\hline \multicolumn{9}{|c|}{ Time Awareness } \\
\hline Time expansion & -0.01 & $\begin{array}{c}{[-0.354} \\
0.289]\end{array}$ & -0.037 & 1 & 0 & $\begin{array}{c}-0.321 \\
0.289]\end{array}$ & 0 & 1 \\
\hline Time pressure & 0 & $\begin{array}{c}{[-0.388} \\
0.296]\end{array}$ & -0.015 & 1 & 0 & {$[-0.316,0.3]$} & -0.025 & 1 \\
\hline \multicolumn{9}{|c|}{ Affect (PANAS Scale) } \\
\hline Positive Affect & 0.133 & $\begin{array}{c}{[-0.222} \\
0.415]\end{array}$ & 0.089 & $<0.001$ & 0.015 & $\begin{array}{c}{[-0.287} \\
0.337]\end{array}$ & 0.036 & 1 \\
\hline Negative Affect & -0.093 & $\begin{array}{c}{[-0.435} \\
0.247]\end{array}$ & -0.071 & 0.052 & 0 & $\begin{array}{c}{[-0.307} \\
0.293]\end{array}$ & -0.031 & 1 \\
\hline \multicolumn{9}{|c|}{ Stress and Well-Being } \\
\hline PSS & -0.081 & $\begin{array}{c}{[-0.413} \\
0.258]\end{array}$ & -0.059 & 0.24 & 0 & $\begin{array}{c}{[-0.316} \\
0.315]\end{array}$ & 0.003 & 1 \\
\hline WHO & 0.105 & $\begin{array}{l}{[-0.17} \\
0.444]\end{array}$ & 0.111 & $<0.001$ & 0.026 & $\begin{array}{c}{[-0.304} \\
0.333]\end{array}$ & 0.053 & 0.728 \\
\hline \multicolumn{9}{|c|}{ Sense of Isolation } \\
\hline Loneliness & -0.057 & $\begin{array}{c}{[-0.342} \\
0.258]\end{array}$ & -0.065 & 0.15 & 0 & $\begin{array}{c}{[-0.333} \\
0.288]\end{array}$ & -0.028 & 1 \\
\hline Distancing & -0.024 & $\begin{array}{c}{[-0.371} \\
0.282]\end{array}$ & -0.044 & 1 & 0 & $\begin{array}{c}{[-0.324} \\
0.316]\end{array}$ & -0.009 & 1 \\
\hline $\begin{array}{c}\text { Personal } \\
\text { Interactions }\end{array}$ & 0.034 & $\begin{array}{c}{[-0.258} \\
0.333]\end{array}$ & 0.057 & 0.322 & 0 & $\begin{array}{c}{[-0.308} \\
0.296]\end{array}$ & 0.024 & 1 \\
\hline $\begin{array}{c}\text { Work and study } \\
\text { interactions }\end{array}$ & 0 & $\begin{array}{c}{[-0.303} \\
0.395]\end{array}$ & 0.032 & 1 & 0 & $\begin{array}{c}{[-0.292} \\
0.293]\end{array}$ & 0 & 1 \\
\hline Personal Care & 0.035 & $\begin{array}{c}{[-0.278} \\
0.321]\end{array}$ & 0.042 & 1 & 0 & $\begin{array}{c}{[-0.307} \\
0.29]\end{array}$ & 0.002 & 1 \\
\hline \multicolumn{9}{|c|}{ Opinion on social distancing } \\
\hline $\begin{array}{l}\text { Opinion on } \\
\text { helping }\end{array}$ & -0.027 & $\begin{array}{c}{[-0.319} \\
0.287]\end{array}$ & -0.036 & 1 & 0 & $\begin{array}{c}{[-0.333} \\
0.335]\end{array}$ & 0.015 & 1 \\
\hline Agreement & 0 & $\begin{array}{c}{[-0.333} \\
0.319]\end{array}$ & -0.001 & 1 & -0.038 & $\begin{array}{c}{[-0.332} \\
0.287]\end{array}$ & -0.028 & 1 \\
\hline News & 0 & $\begin{array}{c}{[-0.289} \\
0.339]\end{array}$ & -0.003 & 1 & -0.02 & $\begin{array}{c}{[-0.361} \\
0.299]\end{array}$ & -0.031 & 1 \\
\hline Self Risk & 0 & $\begin{array}{c}{[-0.316} \\
0.349]\end{array}$ & 0.007 & 1 & 0 & $\begin{array}{c}{[-0.287} \\
0.344]\end{array}$ & 0.032 & 1 \\
\hline Others' Risk & 0 & $\begin{array}{c}{[-0.324} \\
0.258]\end{array}$ & -0.022 & 1 & 0 & $\begin{array}{c}{[-0.272} \\
0.317]\end{array}$ & 0.014 & 1 \\
\hline \multicolumn{9}{|c|}{ Quality of Life } \\
\hline Finances & 0.008 & $\begin{array}{c}{[-0.304} \\
0.341]\end{array}$ & 0.042 & 1 & 0 & $\begin{array}{c}{[-0.344} \\
0.316]\end{array}$ & -0.001 & 1 \\
\hline
\end{tabular}




\begin{tabular}{|c|c|c|c|c|c|c|c|c|}
\hline $\begin{array}{c}\text { Work and study } \\
\text { quality }\end{array}$ & 0 & $\begin{array}{c}{[-0.261} \\
0.357]\end{array}$ & 0.028 & 1 & 0.044 & $\begin{array}{c}{[-0.283} \\
0.374]\end{array}$ & 0.063 & 0.27 \\
\hline Leisure & 0.012 & $\begin{array}{c}{[-0.283} \\
0.394]\end{array}$ & 0.033 & 1 & 0 & $\begin{array}{c}{[-0.294} \\
0.322]\end{array}$ & 0.021 & 1 \\
\hline Family & 0 & $\begin{array}{c}{[-0.333} \\
0.325]\end{array}$ & 0.01 & 1 & 0 & $\begin{array}{c}{[-0.289} \\
0.333]\end{array}$ & 0.032 & 1 \\
\hline Exercises & 0.004 & $\begin{array}{c}{[-0.316} \\
0.334]\end{array}$ & 0.028 & 1 & 0.047 & $\begin{array}{c}{[-0.296} \\
0.359]\end{array}$ & 0.042 & 1 \\
\hline \multicolumn{9}{|c|}{ Routine changes } \\
\hline $\begin{array}{l}\text { Hours of work } \\
\text { and study }\end{array}$ & -0.055 & $\begin{array}{c}{[-0.355} \\
0.316]\end{array}$ & -0.052 & 0.572 & 0 & $\begin{array}{c}{[-0.316} \\
0.289]\end{array}$ & 0.015 & 1 \\
\hline Hours of leisure & 0 & {$[-0.3,0.342]$} & 0.03 & 1 & 0 & $\begin{array}{c}{[-0.281} \\
0.371]\end{array}$ & 0.014 & 1 \\
\hline $\begin{array}{l}\text { Interaction with } \\
\text { housemates }\end{array}$ & 0 & $\begin{array}{l}{[-0.32} \\
0.353]\end{array}$ & 0.018 & 1 & 0 & $\begin{array}{c}{[-0.288} \\
0.338]\end{array}$ & 0.024 & 1 \\
\hline $\begin{array}{l}\text { Hours spent } \\
\text { online }\end{array}$ & 0 & $\begin{array}{c}{[-0.289} \\
0.369]\end{array}$ & 0.026 & 1 & 0 & $\begin{array}{c}{[-0.306} \\
0.316]\end{array}$ & 0.014 & 1 \\
\hline $\begin{array}{l}\text { Hours spent } \\
\text { outside }\end{array}$ & 0.038 & $\begin{array}{c}{[-0.315} \\
0.329]\end{array}$ & 0.053 & 0.572 & 0 & $\begin{array}{c}{[-0.325} \\
0.268]\end{array}$ & 0.002 & 1 \\
\hline Relaxation & 0 & $\begin{array}{c}{[-0.483,} \\
0.474]\end{array}$ & 0.024 & 1 & 0 & {$[-0.5,0.447]$} & 0.02 & 1 \\
\hline
\end{tabular}

Table 5. Correlation of the emotional content of open ended questions and different scales. A Spearman rho was calculated for each participant between the emotional valence of the personal reports and the new and external facts that they shared with the other collected scales. We used a binomial sign test across participants to test whether there was consistent increase/decrease of that measure over the weeks. Cohen's g shows the effect size (proportion of participants with a positive Rho - 0.5) and the respective $p$-value, corrected for multiple comparisons using Holm's method. 


\section{Discussion}

This study investigated time experience during social isolation in response to the COVID-19 pandemic. It was the first reported longitudinal study, where data from a large group of Brazilian participants were collected during 14 weeks to understand how factors such as emotions, stress, feelings of loneliness, and daily routines modulated how participants felt the passage of time. Temporal experience was measured using time awareness scales and the production of temporal intervals. Overall, we found that the production of intervals did not change during social isolation. On the other hand, participants' perceived expansion of time increased, and perceived time pressure decreased at the beginning of social isolation. As the weeks of social isolation went by, the feeling of time expansion seemed to decline, while no strong modulations of time pressure were observed.

Contrary to previous studies that used passage of time judgments (Droit-Volet et al., 2020; Martinelli et al., 2020; R. Ogden, 2021; R. S. Ogden, 2020), we focused on time awareness scales. Specifically, the subjective impression of time before and during social isolation was estimated using scales based on previous research (Wittmann \& Lehnhoff, 2005) that included sentences such as "I often think that time just does not want to pass" and "I haven't enough time to complete my tasks". Our goal, with these scales, was to quantify in more detail participants' time awareness during the weeks. As expected based on previous studies (Wittmann \& Lehnhoff, 2005), two aspects of time awareness emerged from the items when we performed a factor analysis: time expansion (the feeling of empty time) and time pressure (the feeling of time running out).

When asked about their time experience during the first weeks of social isolation, participants reported an increase in the feeling of time expansion (with approximately 65\% of participants reporting this increase) and a decrease in the feeling of time pressure (with approximately $75 \%$ of participants reporting this decrease). These results express the perception of time slowing down during social isolation, in line with previous studies using direct questions about the speed of passage of time (Cellini et al., 2020; Droit-Volet et al., 2020; Martinelli et al., 2020; R. Ogden, 2021; R. S. Ogden, 2020). Interestingly, the results of the present paper and other studies investigating temporal experience during the COVID-19 
pandemic indicate that, even when participants have full access to temporal markers such as clocks (Crawford \& Thor, 1967; Siffre, 1964; Thor \& Crawford, 1964), sunlight and structured daily activities, changes in time awareness are associated with social isolation.

The main drivers of time expansion during social isolation, in our findings, were psychological factors (except age, see below), as evidenced by a strong role of emotions (both the decrease of positive emotions and the increase of negative emotions) and the feeling of loneliness. These results are in line with previous studies that found that the subjective passage of time was affected by the satisfaction of social interactions, decreased task load, increased stress and depression (R. Ogden, 2021; R. S. Ogden, 2020), increased boredom and sadness (Droit-Volet et al., 2020; Martinelli et al., 2020) and a decrease in happiness (Martinelli et al., 2020). Time expansion does not seem to be associated with either demographic (e.g., educational level and income) or objective data on how participants spent this period (where, with whom, with how many people) again in the line of recent studies (Droit-Volet et al., 2020; Martinelli et al., 2020). However, it is important to notice that most of our respondents had a high income and good living conditions during the pandemic, making it impossible to generalize these findings to other, and possibly more difficult, living conditions. Participants' age was associated with time expansion, but more so with younger participants. This effect seems to be more pronounced up to around 40 years old, after which age did not seem to play an important role. This finding is contrary to what was found in studies in the UK and France (Droit-Volet et al., 2020; R. S. Ogden, 2020) in which older participants reported stronger slowing of time, and in the more recent French study that found no effect of age (Martinelli et al., 2020).

For time pressure, the main drivers were more equally divided between objective factors (lower scores on personal care, increased hours of work and study) and psychological factors (higher ratings of perceived stress and higher presence of positive emotions). Previous studies on time during social isolation seem to be more related to our time expansion scores. However, Ogden (R. S. Ogden, 2020) found that increased task load was associated with a feeling of a faster lockdown, consistent with our results of increased hours of work and study being associated with higher time pressure. In general, time pressure seemed to be associated with 
what weighed more in the balance between the cultivation of positive emotions by reserving time for personal care and both the stress of the development of new work dynamics and the danger of contamination.

What could explain such patterns of time awareness during social isolation? According to Zakay (Zakay, 1992), waiting periods are judged based on two central dimensions: temporal relevance (how critical it is that the thing we are waiting for occurs on time) and temporal uncertainty (how certain the timing of the event is). These two factors, in turn, will influence how much attention is given to time (temporal awareness), which will determine how slowly time will seem to pass. Increasing any of these two factors (temporal relevance or uncertainty) will lead to a slowing of time. We found that a general decrease in well-being, especially the absence of positive emotions and higher loneliness levels, was associated with the sensation of time slowing down. These findings are consistent with studies that used experience sampling and found that emotions were the main predictor of passage of time judgements (Droit-Volet \& Wearden, 2016, 2015).

Positive emotions have been associated with better coping mechanisms, i.e., self-regulation skills, which are essential for the psychological management of crises (Cohn et al., 2009; Fredrickson et al., 2003). Time studies in which participants had to wait with no sources of distraction during 7.5 minutes showed that people who were less able to emotionally self-regulate in daily life and were more impulsive felt time passing slower (Jokic et al., 2018; Witowska et al., 2020). It is possible that people showing lower levels of positive emotions also had trouble in dealing with social isolation by themselves. The lack of internal resources could direct them to wait on external agents to ameliorate the situation, increasing the temporal relevance of the end of social isolation. Concurrently, the country's political and sanitary situation during the sampled period was very troubled, with possible impacts in temporal uncertainty.

Although the temporal awareness approach proposed by Zakay (Zakay, 1992) fits well with our findings, this model was not initially designed to explain the passage of time judgements. The majority of cognitive models on perception of time distinguish two fundamental perspectives: prospective and retrospective timing. While prospective timing 
refers to the judgment of duration that is presently being experienced (i.e., our time production task), retrospective time perception refers to the judgment of a time interval that has already elapsed (Block \& Zakay, 1997). Recently, the term 'passage of time judgments' was also suggested for phenomena that do not seem to correspond with either prospective or retrospective duration judgments (Jones, 2019; Wearden, 2005), but instead with the feeling of how quickly time seems to pass. Whether and how retrospective and passage of time judgements are correlated is still under debate (Jones, 2019; Wearden, 2005). Future studies, investigating the perceived retrospective duration of the period of social isolation can bring important advances on how these different abilities relate.

We also measured the production of time intervals in the seconds range and did not find correlations between produced intervals and time awareness. Previous studies have suggested that time awareness (as measured by scales and passage of time judgments) and temporal durations judgments (measured by timing tasks) are not directly related (Droit-Volet \& Wearden, 2016). On the other hand, whether levels of depression and anxiety can modulate temporal judgments is still not clear, with mixed and inconclusive findings (Thönes \& Oberfeld, 2015; Wittmann, 2009). Our study used intervals from a wide range (of 1, 3, and 12s) to investigate whether potential effects of psychological factors on production of time intervals depended on the specific duration of these intervals (Thönes \& Oberfeld, 2015; Wittmann, 2009) but we did not find consistent results for any of the tested durations. Although not necessarily surprising, these results are important in that they suggest that time estimation models can not account for the effects of social isolation on time awareness.

Our study was, to the best of our knowledge, the first to examine changes in participants' responses throughout social isolation (during 14 weeks). Given that our first session took place approximately two months since the beginning of social isolation in Brazil, the results of the first session might still reflect how participants reacted to their new living conditions and to their initial impressions of time experience. In fact, reports of time expansion had a small decrease during the weeks of social isolation, while no significant decrease/increase was found for time pressure. Tracking the same participants throughout the weeks of social isolation allowed us to investigate how changes in subjective and objective measures are 
reflected in their time awareness. In general, there was a strong consistency between the most important predictors for both time awareness scales comparing the results of the first session with the results of the weekly sessions. The main predictors for time expansion were psychologically oriented, such as the absence of positive emotions, loneliness, and well-being. For time pressure, there was a balanced contribution between psychological factors (perceived stress and personal care) and more objective factors (how much and how satisfactory were the work/study interactions). These findings show that these factors modulate time awareness not only across participants, but also within participants, with weekly fluctuations of these measures associated with concurrent modulations in time experience.

In addition to trying to understand how time experience changed during social isolation, our results also allowed us to observe how different scales' evolved during this period and how these changes might influence time awareness. For example, we found a widespread improvement of well-being throughout the weeks of social isolation, with decreased stress levels and negative emotions, and increased well-being. Critically, during this period, Brazil's COVID-19 cases increased and, only towards the last weeks of our research, started to stabilize. Still, participants seemed to adapt to their new conditions, as can be seen in the general increase in quality of life measures and positive changes in participants' routines. In general, there was a tendency that seemed to go back to pre-isolation patterns. Participants reported spending more time outside, less time online, and increasing their time dedicated to leisure. We also observed a general decrease in how much participants felt that their social isolation helped and increased the sense of being at risk. Finally, we found consistent changes in their routines during the 14 weeks of social isolation. However, the improvements in daily routines were not followed by substantial concomitant changes in time awareness. This dissociation highlights, once again, how time awareness is more related to how participants are experiencing the period of social isolation than to concrete changes in their routine.

In conclusion, we found that social distancing had substantial effects on temporal experience. These effects were more strongly observed in participants' time awareness but not in their ability to produce temporal intervals. The factors more associated with time slowing down both across participants and within participants were the absence of positive emotions 
and loneliness. The feeling of higher time pressure was modulated by the balance between stress levels and reserving time for personal care. Taken together, our findings show how emotions are a crucial aspect of how time is felt. 


\section{Methods}

\section{Procedure}

The National Research Ethics Commission (CONEP) approved the experimental protocol (CAEE: 30413420.3.0000.0071), and the experiment was performed following the approved guidelines and regulations. Participants completed online questionnaires distributed through

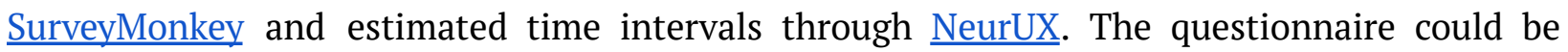
completed either on computer or cell phone. We developed two similar questionnaires, one that participants filled in their first session and a second to be completed every week. Translated and original surveys can be found at https://osf.io/u783q/.

\section{First Session Protocol}

Participants were recruited through social media and email lists. The questionnaire was released to participants on the 6th of May, 2020. In Brazil, social isolation protocols were uncentralized from the Federal government and thus varied across regions in their implementation dates and restrictiveness. Most states started applying these protocols around the 16th of March, 2020. Participants were able to join the study by performing the first session until the 17th of June (6 weeks after we started distributing the survey).

The first session started with participants completing a registration form at $\underline{\text { NeurUX}}$, in which they filled their name and contact information (telephone number and email). This information was necessary so that each participant was associated with an unique ID and their responses could be combined throughout the sessions. Also, this personal information was kept in a separate database, with only the ID number being saved into the time estimation and survey databases. Participants were then asked to estimate temporal intervals of 1,3 , and $12 \mathrm{~s}$ by pressing a button to start the trial and another button to stop when they thought the interval had passed. Each interval was prompted three times in random order. The interval to be estimated was displayed on the computer or smartphone screen, which also contained two buttons: a start and a stop. We provided no feedback on their performance. When the estimations were done, participants were redirected to the survey page. The software was 
developed in JavaScript by NeurUX enterprise, which also handled the redirection of participants to the right survey every session.

\section{$\underline{\text { Measures }}$}

Participants completed the following questionnaires in this order:

Demographic questions: Participants stated their age, gender, profession, state of residence, education level, and average monthly income.

Social isolation situation: Number of days in isolation, where they were staying during the pandemic, housing conditions (number of private indoor/outdoor areas), number of housemates, number of children or teenagers as housemates, the existence of a personal private room, and pet ownership.

COVID-19 related questions: Participants responded whether: i) themselves, somebody of their family or someone they knew had COVID-19 related symptoms; ii) themselves, somebody of their family or someone they knew had been hospitalized due to COVID-19; iii) somebody of their family or someone they knew had passed away due to COVID-19.

Time awareness scales: Time awareness scales were based on previous work investigating subjective time perception (Wittmann \& Lehnhoff, 2005). The scales consisted of statements on the personal experience of time to which participants stated how much they agreed on using a visual analogue scale (VAS, 101 points, ranging from 0 to 100) with the anchors "not at all” and "I strongly agree". Scales focused on two different aspects of temporal experience: Time Expansion and Time Pressure. In the first session, participants completed both scales twice, once relative to their experience of time before the social isolation period and the other relative to their experience since the beginning of social isolation. The time awareness scales consisted of the following statements: 
Time Expansion (relative to the period before the social isolation/relative to the period during the social isolation):

1. I felt that my time seemed empty.

2. I often felt that time just did not want to pass.

3. I often felt bored.

4. I often felt I had a lot of time.

5. I often felt that I spent my time without doing anything.

Time Pressure (relative to the period before the social isolation/relative to the period during the social isolation):

1. I felt I did not have enough time to complete my tasks.

2. I often felt time pressure.

3. I often felt I did have enough time to devote myself to important things.

4. I often felt that time was running out.

5. I felt that I had to establish my priorities because I could not do everything I had to do.

Sense of isolation: This scale consisted of five questions in which participants reported how much the following feelings decreased or increased after their social isolation (VAS, 101 point scale, ranging from -50 to 50 with anchors "Decreased" and "Increased"): i) loneliness; ii) social distancing; iii) quality of their social interactions; iv) quality of their interactions in the work and/or study environment; v) self-care (personal time).

Opinion on the social isolation protocol: Participants stated how much they agreed on the following views about the Brazilian social isolation protocol for controlling COVID-19 (VAS, 101 point scale, ranging from -50 to 50 with anchors "Not at all" and "I strongly agree"): i) I feel my isolation is helping to contain the disease; ii) I am in favour of the social isolation measures adopted so far; iii) I have been searching and following the news about the disease; iv) I feel I am at risk of contamination; v) I feel people who I care about are at risk of contamination. 
Changes in the quality of life: Participants reported how much they felt that the following aspects had worsened or improved during social isolation (VAS, 101 point scale, ranging from -50 to 50 with anchors "Worsened" and "Improved"): i) finances; ii) work and/or study routine; iii) leisure time; iv) family time; v) exercise routine.

Changes in the routine related to the activity hours: Participants reported whether there was a decrease/increase during social isolation on the hours spent (VAS, 101 point scale, ranging from -50 to 50 with anchors "Decreased" and "Increased"): i) face-to-face work and/or study; ii) face-to-face social meetings; iii) interacting with housemates; iv) online interactions; v) outdoor activities; after their social isolation.

The Positive and Negative Affect Schedule (PANAS): To evaluate affect, participants responded to a validated version of the PANAS in Brazilian Portuguese (Carvalho et al., 2013). This questionnaire provides independent indexes of positive and negative affect by considering 9-item scales (active, alert, attentive, determined, enthusiastic, excited, inspired, interested, strong) for the positive and 10-item scales (afraid, ashamed, distressed, guilty, hostile, irritable, jittery, nervous, scared, upset) for the negative. The "proud" item was included in the assessment but not considered in the positive index calculation because the translated word for proud is ambiguous (Carvalho et al., 2013) in Brazilian Portuguese, likely evoking a pejorative connotation. Participants responded either slightly/not at all (points $=1$ ); a little (points $=2$ ); moderately (points $=3$ ); quite a bit (points $=4$ ); or extremely (points $=5$ ) to the items.

Perceived stress scale (PSS-10): The perception of respondents' stress was evaluated by the PSS-10 (S. Cohen et al., 1983) adapted to Brazilian Portuguese (Luft et al., 2007). This 10-item scale measures how much participants perceive their lives as unpredictable, uncontrollable, and overwhelming. Participants answered never (points $=0$ ), almost never (points $=1$ ), sometimes (points $=2$ ), fairly often (points $=3$ ), very often (points $=4$ ) to positive and negative statements. The PSS score was obtained by reversing the four positive items (items 4, 5, 7, \& 8) and then summing the responses to all items (Luft et al., 2007). 
World Health Organization 5-item well-being index (WHO-5): Well-being was assessed by using the WHO-5 scale in Brazilian Portuguese (World Health Organization; de Souza \& Hidalgo, 2012). Participants answered never (points $=0$ ), sometimes (points $=1$ ), less than half of the time (points = 2), more than half of the time (points = 3), most of the time (points =4), and all the time (points $=5$ ) to the frequency of occurrence of positively worded statements at this short 5-item scale.

Open Questions: At the end of the survey, participants were invited to complete two open questions. We asked them to (1) briefly share the external facts (news, events) that have marked them most since the beginning of the isolation; and (2) report what has most marked them personally since the beginning of the isolation. Both questions were non-obligatory with an open limit of characters.

\section{Weekly Sessions Protocol}

Every Thursday, participants were invited to complete a weekly session, which they had until the next Thursday to complete. These weekly sessions consisted of the temporal estimation procedure and the following questions/scales: COVID-19 related questions; Time Expansion; Time Pressure; Sense of isolation; Opinion on social isolation protocol; Changes in the quality of life; Changes in the routine related to the activity hours; PANAS; PSS-10; WHO-5; and open questions. These sessions focused on comparisons between the current and last week. This differs from the first session questionnaire, which approached the comparison between the pre and post isolation periods.

Starting in the seventh week (June 18th), all surveys included a new question, asking about participants' current level of social isolation. This question was added due to the easing of restrictions on social isolation measures in Brazil at that time. In this new question, we asked: "Regarding your isolation level during last week". Participants responded through a VAS with the extreme values at "my activities are home-restricted" to "I totally returned to my pre-isolation routine." 
Surveys were collected until August 21st, 2020. Therefore, participants were invited to complete 15 surveys (the first session and 14 weekly sessions).

\section{Data Analyses}

All data analyses were performed using R (R Core Team, 2020; version 4.0.2, (Computing \& Others, 2013) and Python. Statistical analysis and effect sizes were performed in Python using the Pingouin library, v.0.3.8 (Vallat, 2018). Statistical analysis scripts are open and available at https://osf.io/u783q/.

Given that the majority of our variables were based on scales, most of the reported statistical analyses are based on non-parametric tests (Kruskal-Wallis, Friedman test for repeated measurements and non-parametric correlations). When necessary, p-values from multiple comparisons were corrected using the Holm method. When available, effect sizes are reported using the common language effect size-CLE (McGraw \& Wong, 1992; Vargha \& Delaney, 2000) as implemented in the Pingouin library. The common language effect size is the proportion of pairs in which data from sample $x$ is higher than data from sample $y$.

For analysis investigating the correlation between variables in the first session, we used either Spearman correlation or the Shepherd pi correlation (as implemented in the Pingouin library), a robust method that returns the Spearman correlation coefficient after removing bivariate outliers (Rousselet \& Pernet, 2012; Schwarzkopf et al., 2012). Similar results were obtained when using other robust methods, such as Skipped correlation.

When investigating monotonic increases/decreases of responses as a function of week, we performed a two-stage analysis. First, we estimated the Spearman coefficients between week and the measure of interest (e.g., time expansion, time pressure, report polarity) per participant. Then, the estimated coefficients were compared to zero using a binomial sign-test. Effect sizes for the sign-test were estimated using Cohen's g as suggested in Rosnow and Rosenthal (2003). 


\section{Preprocessing and exclusions}

The first survey had 4388 registrations. We removed data from participants that did not complete the full questionnaire $(\mathrm{n}=523,12 \%)$ and participants' IDs that were doubled (due to connection problems, $\mathrm{n}=10,0.002 \%$ ). Our final sample for session 1 was of 3855 participants. For the weekly surveys, we removed all data from participants that did not complete the full questionnaire and participants that did not complete session 1. All analysis on the weekly sessions were performed on data from participants that completed at least 4 weekly sessions ( $25 \%$ of the sessions), for a total of 953 participants.

\section{$\underline{\text { Temporal estimates }}$}

Participants produced three temporal intervals of 1,3 and 12 s, summing a total of nine estimates requested in random order. Estimates lower than 0.25 and greater than $48 \mathrm{~s}$ were removed, and the remaining productions were averaged according to the requested interval. Participants were excluded if all their reproductions of a single interval were considered outliers $(n=5)$. The median absolute deviation (MAD) was calculated in the aggregate of participants' log-transformed estimates separately for each interval. Participants whose average estimates were three deviations below or above the MAD in any interval were considered outliers $[1 \mathrm{~s}$ range $=(0.37,4.81), 3 \mathrm{~s}$ range $=(0.95,9.07), 12 \mathrm{~s}$ range $=(3.08,37.91), \mathrm{n}=$ 255] and excluded from further analysis (Leys et al., 2013; Rousseeuw \& Croux, 1993). The final sample for temporal estimates analysis was of 3595 participants in the first session. Time estimation values were logarithmically transformed only for MAD analysis and for graphical purposes.

A similar procedure was used in the weekly sessions. Estimates lower than 0.25 or greater than 48 s were excluded, and once more participants were excluded if all reproductions of a single interval were considered outliers $(n=4)$. We used three deviations of MAD calculated in the aggregate of log-transformed estimates for each interval to exclude outliers, causing the indirect exclusion of participants $[1 \mathrm{~s}$ range $=(0.35,4.35), 3 \mathrm{~s}$ range $=(0.87,8.89), 12 \mathrm{~s}$ range $=$ (2.44, 40.98), $\mathrm{n}=157]$. The final sample for temporal estimates descriptive analysis was of 3694 participants in the weekly sessions. Only participants with four sessions or more were included 
in the regression analyzes $(n=927)$. As for the first session estimations, the weekly time estimations were logarithmically transformed only for MAD analysis and graphical purposes.

\section{Validated scales (PSS-10, WHO-5, PANAS)}

The scores for the PSS-10 (Luft et al., 2007), WHO-5 (World Health Organization; de Souza \& Hidalgo, 2012) and PANAS (Carvalho et al., 2013) were calculated according to their respective Brazilian validations. In the first session, all scales showed adequate reliability indices [McDonald's Omega (CI 95\%): PSS-10 $=0.87(0.63,0.87)$, WHO-5 $=0.89(0.85,0.93)$, PANAS $=0.91$ (0.87, 0.97); Cronbach's Alpha (CI 95\%): PSS-10 = $0.87(0.57,0.88)$, WHO-5 = 0.89 $(0.80,0.92)$, PANAS $=0.88(0.77,0.95)]$.

\section{Custom scales}

Four scales were created to measure different impacts of social isolation: Sense of isolation, Opinion on the social isolation protocol, Changes in the quality of life, Changes in the routine related to the activity hours. These scales were developed to include items that made direct reference to the unprecedented situation of the COVID-19 pandemic. To decide whether the items of these scales should be added independently as features in our machine learning algorithm, or if subsets of the items were measuring a latent construct, we used an exploratory factor analysis approach and measured the reliability of each scale. This analysis was performed on the responses of the first session, in which there were a higher number of participants. All scales showed poor factor adequacy, precluding the use of factor analysis models (KMOs < 0.66). Furthermore, the reliability of the scales was also low (McDonald's Omegas < 0.66; Cronbach's Alphas $<0.64$ ), indicating that the scales were not unidimensional. In face of these psychometric characteristics, raw scores were used as features.

\section{$\underline{\text { Time awareness scales }}$}

Given that the time awareness scales were used in Brazilian Portuguese for the first time, we investigated their construct validity with factor analyses. We divided the first session data into even-odds partitions for exploratory and confirmatory analyses $\left(\mathrm{N}_{\text {even }}=1928 ; \mathrm{N}_{\text {odds }}=\right.$ 
1927). We expected our scale to behave similarly to the original scale in English, so in our exploratory approach, we started fitting a maximum likelihood confirmatory model with two correlated factors: Time Expansion and Time Pressure. Then, modification indexes were inspected and modifications regarding cross-loadings for bad indicators (loadings $<0.6$ ) were added. If the indicator still performed poorly, it was removed from the analysis. After obtaining a robust model with the exploratory approach (see openly available code), we applied the same model to the even partition of the data in a strict confirmatory approach (i.e. without making any modifications to improve fit). These procedures were applied separately for the data from the scales with phrasings regarding the period pre-isolation and during isolation.

Time awareness scales in Brazilian Portuguese showed good construct validity, as assessed by factor analyses. The factors Time Expansion and Time Pressure clearly emerged from the proposed items (all retained items loaded in one factor with loading above 0.6 ). Only one item in the Time Expansion subscale ("I often felt I had a lot of time") was a poor indicator and was dropped. These results held for both pre and during isolation item phrasings [pre-isolation: chi-squared $(26)=156.40, \mathrm{p}<0.001 ; \mathrm{CFI}=0.99 ; \mathrm{AGFI}=0.97 ;$ RMSEA $(\mathrm{CI} 95 \%)=$ 0.05 (0.04, 0.06); during isolation: chi-squared $(26)=362.60, \mathrm{p}<0.001 ; \mathrm{CFI}=0.97$; AGFI $=$ 0.93; RMSEA $(\mathrm{CI} 95 \%)=0.08(0.08,0.09)]$. Factors' correlation was non-significant for the pre-isolation phrasing ( $r$ ho $=-0.02, \mathrm{p}=0.37$ ). The during isolation phrasing results showed a low negative correlation (rho $=-0.21, \mathrm{p}<0.001$ ). Reliability measures were also good for both phrasings [pre-isolation: McDonald's Omega $(\mathrm{CI} 95 \%)=0.91(0.76,0.97)$; Cronbach's Alpha (CI $95 \%)=0.83(0.41,0.95)$, during isolation: McDonald's Omega $($ CI 95\%) $=0.92(0.92,0.98)$; Cronbach's Alpha $($ CI 95\% $)=0.86(0.86,0.97)]$.

\section{Natural language processing}

For both personal and external reports, we analysed the emotional polarity content. To have a common standard, we performed a normalization that removed emojis, HTML tags, URLs, twitter-like mentions, hashtags, enumerations, and white-spaces between words. We translated all sentences to English using a Google Translate API for Python (https://pypi.org/project/googletrans/). To classify the polarity of the sentence we used the 
Stanford Sentiment Treebank (Socher et al., 2013) wrapped in Stanza library (Qi et al., 2020). All default values suggested on Stanza library were maintained, however differently from the Java version, we cannot prevent the model from breaking-line on each period, so we changed all reports' periods to semicolons. Finally, we performed the SST prediction obtaining 5 categories of polarities (very-negative, negative, neutral, positive, and very-positive).

Given that the SST is originally trained in native English and we used it in translated sentences, we aimed to validate this pipeline. To do so, we used the Brazilian Portuguese Corpus ReLi (Cláudia Freitas, Eduardo Motta, R Milidiú, Juliana César, 2012) that consists of seven book reviews - texts with one or more sentences - with manual-annotation and sentence-level polarity (negative, neutral, and positive). Because the Corpus ReLi labels all words of a sentence with the same polarity, we split all reviews into sentences and excluded any sentences with words of different polarities. Then, we translated all sentences and classified them using the SST, the same strategy used with participants' reports. Given that ReLi labels are classified only into three levels (negative, neutral, and positive), we used a similar classification for the SST, with both "very negative" and "negative" being classified as negative and "positive" and "very positive" as positive. To evaluate the classification using this pipeline, we estimated both the accuracy of the SST predictions (proportion correct $=0.45$, binomial test against 0.33, p<0.001) and a quadratic weighted Cohen's Kappa (kappa=0.248, p<0.001) (J. Cohen, 1960) as included on the pyirr package (https://pypi.org/project/pyirr/).

For the first session reports, a Shepherd correlation was performed between the polarities and all other scales scores. All p-values obtained were corrected for multiple comparisons using Holm's method. For weekly sessions reports, a Spearman correlation was performed between polarities and all others weekly measures for each participant. At the group level, a sign-test with corrections for multiple comparisons using Holm's method was used.

\section{Exploratory data analysis and hierarchical clustering}

A hierarchical clustering using a bottom-up approach was applied to the correlation between scales $($ distance $=1-\mid$ rho|). This method performs a hierarchical clustering in which each scale starts in its cluster, and clusters are successively merged. The metric used for the merge strategy was the complete linkage, which minimizes the maximum distance between 
observations of pairs of clusters. For display purposes, distances associated with a medium effect size $(\mid$ rho $\mid \geqslant 0.3)$ were colored using the same color.

For the weekly exploratory data analysis (Figure 6), the pairwise correlation between weekly scales was calculated separately per participant. At the group level, the median rho for each pairwise correlation across participants was calculated. The hierarchical clustering was similar to that of session 1.

\section{Machine Learning}

All machine learning models used to understand the relationship between time awareness scales and other factors used the Catboost method (Dorogush et al., 2018; Prokhorenkova et al., 2018), an open-sourced gradient boosting library as implemented in https://github.com/catboost. Catboost uses a gradient boosting method that can capture linear and non-linear relations between different features and the score of interest. Given that the majority of features and dependent variables were bounded scores, linear methods are not ideal for this kind of prediction. For this reason, we opted to use an ensemble-based method. Among ensemble methods, Catboost has performance similar to other algorithms such as LightGBM and XGBoost. However, one of the main advantages of Catboost is that it achieves good performance using default hyper-parameters, with no need to tune them. All analyses reported here used the default parameters in the CatboostRegressor methods, version 0.23.2

Models were trained and tested using five-fold cross-validation over the dataset. For the weekly sessions, a GroupKFold (with $\mathrm{K}=5$ ) with non-overlapping groups was used. This variant guarantees that data of a given participant will not appear in two different folds, avoiding that the same participant is included both at the training and test sets in a given iteration.

Model performance was evaluated by estimating the coefficient of determination (R2) between predicted and observed values. This coefficient may have a maximum value of 1 for a perfect fit and a minimum value of zero or even negative values (when the mean of the data provides a better fit than the predicted values). The R2 was estimated on the test set of each fold and averaged across folds for each model. To estimate how stable performance was, we fit 500 iterations of the model and report the median, minimum, and maximum R2. For session 1, these 500 iterations used different 5-fold separations. For the weekly sessions, a group shuffle 
split was used in which, in each iteration, $20 \%$ of the participants were allocated on the test set and the other $80 \%$ on the training set.

To evaluate model performance, we also calculated a permutation test score, which represents how likely an observed performance of the classifier would be obtained by chance. To generate the null distribution of performance, the R2 metric was measured in 500 different permutations of the dataset. In each permutation the labels (the time expansion or time pressure score) of the training set were randomly shuffled, thereby removing any dependency between the features and the labels. Finally, performance was also compared with a baseline model, with a smaller number of features. For session 1, the features included in the baseline model were restricted to time expansion and time pressure in the period before social isolation. For the weekly sessions, the baseline model consisted of time expansion and time pressure in the period before social isolation and week.

To interpret the model's decisions, we used SHAP (SHapley Additive exPlanations), a unified approach that connects cooperative game theory with local explanations to explain any machine learning model (Scott M. Lundberg et al., 2020; S. M. Lundberg \& Lee, 2017). The SHAP value shows the average contribution of a feature value to the model's prediction, or, in other words, given a current set of feature values, the contribution of a feature value to the difference between the actual prediction and the mean prediction (Molnar, 2019). The goal of SHAP is to explain the prediction of an instance $\mathrm{x}$ by computing the contribution of each feature to the prediction, using Shapley values. SHAP estimates from the Catboost algorithm were estimated using internal functions of the Catboost library and were always estimated on the test set. We report and show the SHAP values calculated for a single model. However, to assess how stable SHAP values were across different iterations and folds, supplemental figures in OSF show the distribution of SHAP values across 500 iterations. As shown, SHAP values and the most relevant features were highly consistent across iterations. 


\section{References}

Allen, M., Poggiali, D., Whitaker, K., Marshall, T. R., \& Kievit, R. A. (2019). Raincloud plots: a multi-platform tool for robust data visualization. Wellcome Open Research, 4, 63.

Block, R. A., \& Zakay, D. (1997). Prospective and retrospective duration judgments: A meta-analytic review. Psychonomic Bulletin \& Review, 4(2), 184-197.

Carvalho, H. W. de, Andreoli, S. B., Lara, D. R., Patrick, C. J., Quintana, M. I., Bressan, R. A., Melo, M. F. de, Mari, J. de J., \& Jorge, M. R. (2013). Structural validity and reliability of the Positive and Negative Affect Schedule (PANAS): Evidence from a large Brazilian community sample. Brazilian Journal of Psychiatry, 35(2), 169-172.

Cellini, N., Canale, N., Mioni, G., \& Costa, S. (2020). Changes in sleep pattern, sense of time and digital media use during COVID-19 lockdown in Italy. Journal of Sleep Research, 29(4), e13074.

Cláudia Freitas, Eduardo Motta, R Milidiú, Juliana César. (2012). Vampiro que brilha... rá! Desafios na anotaçao de opiniao em um corpus de resenhas de livros. XI Encontro de Linguística de Corpus.

https://www.linguateca.pt/Repositorio/ReLi/Anais_ELC2012_Freitasetal.pdf

Cohen, J. (1960). A Coefficient of Agreement for Nominal Scales. In Educational and Psychological Measurement (Vol. 20, Issue 1, pp. 37-46). https://doi.org/10.1177/001316446002000104

Cohen, S., Kamarck, T., \& Mermelstein, R. (1983). A Global Measure of Perceived Stress. Journal of Health and Social Behavior, 24(4), 385.

Cohn, M. A., Fredrickson, B. L., Brown, S. L., Mikels, J. A., \& Conway, A. M. (2009). Happiness 
unpacked: positive emotions increase life satisfaction by building resilience. Emotion , 9(3), $361-368$.

Computing, R., \& Others. (2013). R: A language and environment for statistical computing. $R$ Core Team.

https://www.yumpu.com/en/document/view/6853895/r-a-language-and-environment-for-s tatistical-computing

Crawford, M. L., \& Thor, D. H. (1967). Time perception in children in the absence of external temporal synchronizers. Acta Psychologica, 26(2), 182-188.

Dorogush, A. V., Ershov, V., \& Gulin, A. (2018). CatBoost: gradient boosting with categorical features support. In $\operatorname{arXiv}$ [cs.LG]. arXiv. http://arxiv.org/abs/1810.11363

Droit-Volet, S., Fayolle, S., Lamotte, M., \& Gil, S. (2013). Time, Emotion and the Embodiment of Timing. Timing \& Time Perception, 1(1), 99-126.

Droit-Volet, S., Gil, S., Martinelli, N., Andant, N., Clinchamps, M., Parreira, L., Rouffiac, K., Dambrun, M., Huguet, P., Dubuis, B., Pereira, B., COVISTRESS network, Bouillon, J.-B., \& Dutheil, F. (2020). Time and Covid-19 stress in the lockdown situation: Time free, «Dying» of boredom and sadness. PloS One, 15(8), e0236465.

Droit-Volet, S., \& Wearden, J. (2016). Passage of Time Judgments Are Not Duration Judgments: Evidence from a Study Using Experience Sampling Methodology. Frontiers in Psychology, 7, 176.

Droit-Volet, S., \& Wearden, J. H. (2015). Experience Sampling Methodology reveals similarities in the experience of passage of time in young and elderly adults. Acta Psychologica, 156, 77-82. 
Fredrickson, B. L., Tugade, M. M., Waugh, C. E., \& Larkin, G. R. (2003). What good are positive emotions in crisis? A prospective study of resilience and emotions following the terrorist attacks on the United States on September 11th, 2001. In Journal of Personality and Social Psychology (Vol. 84, Issue 2, pp. 365-376). https://doi.org/ 10.1037/0022-3514.84.2.365

Jokic, T., Zakay, D., \& Wittmann, M. (2018). Individual Differences in Self-Rated Impulsivity Modulate the Estimation of Time in a Real Waiting Situation. Timing \& Time Perception, 6(1), 71-89.

Jones, L. A. (2019). The Perception of Duration and the Judgment of the Passage of Time. In V. Arstila, A. Bardon, S. E. Power, \& A. Vatakis (Eds.), The Illusions of Time: Philosophical and Psychological Essays on Timing and Time Perception (pp. 53-67). Springer International Publishing.

Leys, C., Ley, C., Klein, O., Bernard, P., \& Licata, L. (2013). Detecting outliers: Do not use standard deviation around the mean, use absolute deviation around the median. In Journal of Experimental Social Psychology (Vol. 49, Issue 4, pp. 764-766).

https://doi.org/10.1016/j.jesp.2013.03.013

Locsin, R. C. (1993). Time Experience of Selected Institutionalized Adult Clients. In Clinical Nursing Research (Vol. 2, Issue 4, pp. 451-463).

https://doi.org/10.1177/105477389300200407

Luft, C. D. B., Sanches, S. de O., Mazo, G. Z., \& Andrade, A. (2007). [Brazilian version of the Perceived Stress Scale: translation and validation for the elderly]. Revista de saude publica, $41(4), 606-615$.

Lundberg, S., \& Lee, S.-I. (2017). A Unified Approach to Interpreting Model Predictions. In 
$\operatorname{arXiv}$ [cs.AI]. arXiv. http://arxiv.org/abs/1705.07874

Lundberg, S. M., Erion, G., Chen, H., DeGrave, A., Prutkin, J. M., Nair, B., Katz, R., Himmelfarb, J., Bansal, N., \& Lee, S.-I. (2020). From Local Explanations to Global Understanding with Explainable AI for Trees. Nature Machine Intelligence, 2(1), 56-67.

Lundberg, S. M., \& Lee, S. I. (2017). A unified approach to interpreting model predictions. Advances in Neural Information Processing Systems, 4765-4774.

Martinelli, N., Gil, S., Belletier, C., Chevalère, J., Dezecache, G., Huguet, P., \& Droit-Volet, S. (2020). Time and Emotion During Lockdown and the Covid-19 Epidemic: Determinants of Our Experience of Time? Frontiers in Psychology, 11, 616169.

McGraw, K. O., \& Wong, S. P. (1992). A common language effect size statistic. In Psychological Bulletin (Vol. 111, Issue 2, pp. 361-365). https://doi.org/ 10.1037/0033-2909.111.2.361

Molnar, C. (2019). Interpretable Machine Learning. Lulu.com.

Ogden, R. (2021). Distortions to the passage of time during England's second national lockdown: A role for depression. PloS One, 16(4), e0250412.

Ogden, R. S. (2020). The passage of time during the UK Covid-19 lockdown. PloS One, 15(7), e0235871.

Prokhorenkova, L., Gusev, G., Vorobev, A., Dorogush, A. V., \& Gulin, A. (2018). CatBoost: unbiased boosting with categorical features. In S. Bengio, H. Wallach, H. Larochelle, K. Grauman, N. Cesa-Bianchi, \& R. Garnett (Eds.), Advances in Neural Information Processing Systems (Vol. 31, pp. 6638-6648). Curran Associates, Inc.

Qi, P., Zhang, Y., Zhang, Y., Bolton, J., \& Manning, C. D. (2020). Stanza: A Python Natural Language Processing Toolkit for Many Human Languages. http://arxiv.org/abs/2003.07082 
Rosnow, R. L. (2003). Effect sizes for experimenting psychologists. Canadian Journal of Experimental Psychology = Revue Canadienne de Psychologie Experimentale, 57(3), 221-237.

Rousseeuw, P. J., \& Croux, C. (1993). Alternatives to the Median Absolute Deviation. In Journal of the American Statistical Association (Vol. 88, Issue 424, pp. 1273-1283). https://doi.org/10.1080/01621459.1993.10476408

Rousselet, G. A., \& Pernet, C. R. (2012). Improving standards in brain-behavior correlation analyses. Frontiers in Human Neuroscience, 6, 119.

Schwarzkopf, D. S., De Haas, B., \& Rees, G. (2012). Better ways to improve standards in brain-behavior correlation analysis. Frontiers in Human Neuroscience, 6, 200.

Siffre, M. (1964). Beyond time. London: Chatto and Windus.

Socher, R., Perelygin, A., Wu, J., Chuang, J., Manning, C. D., Ng, A. Y., \& Potts, C. (2013). Recursive Deep Models for Semantic Compositionality Over a Sentiment Treebank. Proceedings of the 2013 Conference on Empirical Methods in Natural Language Processing, $1631-1642$.

Thönes, S., \& Oberfeld, D. (2015). Time perception in depression: a meta-analysis. Journal of Affective Disorders, 175, 359-372.

Thor, D. H., \& Crawford, M. L. J. (1964). Time perception during a two-week confinement: Influence of age, sex, IQ, and time of day. Acta Psychologica, 22, 78-84.

Vallat, R. (2018). Pingouin: statistics in Python. Journal of Open Source Software, 3(31), 1026. Vargha, A., \& Delaney, H. D. (2000). A Critique and Improvement of the CL Common Language Effect Size Statistics of McGraw and Wong. In Journal of Educational and Behavioral Statistics (Vol. 25, Issue 2, pp. 101-132). https://doi.org/ 10.3102/10769986025002101 
Wearden, J. H. (2005). The wrong tree: time perception and time experience in the elderly. In Measuring the Mind: Speed, control, and age (pp. 137-158). Oxford University Press.

Witowska, J., Schmidt, S., \& Wittmann, M. (2020). What happens while waiting? How self-regulation affects boredom and subjective time during a real waiting situation. Acta Psychologica, 205, 103061.

Wittmann, M. (2009). The inner experience of time. Philosophical Transactions of the Royal Society of London. Series B, Biological Sciences, 364(1525), 1955-1967.

Wittmann, M. (2020). Subjective Passage of Time during the Pandemic: Routine, Boredom, and Memory. KronoScope, 20(2), 260-271.

Wittmann, M., \& Lehnhoff, S. (2005). Age Effects in Perception of Time. In Psychological Reports (Vol. 97, Issue 3, pp. 921-935). https://doi.org/ 10.2466/pr0.97.3.921-935

Zakay, D. (1992). On Prospective Time Estimation, Temporal Relevance and Temporal Uncertainty. In F. Macar, V. Pouthas, \& W. J. Friedman (Eds.), Time, Action and Cognition: Towards Bridging the Gap (pp. 109-117). Springer Netherlands.

de Souza, C.M., Hidalgo, M.P.L. World Health Organization 5-item well-being index: validation of the Brazilian Portuguese version. Eur Arch Psychiatry Clin Neurosci 262, 239-244 (2012).

World Health Organization WHO-Five well-being index-background information. http://www.who-5.org/ 


\section{Authors' contributions}

\begin{tabular}{|c|c|c|c|c|c|c|c|c|c|c|c|}
\hline & $\begin{array}{c}\text { André } \\
\text { M. } \\
\text { Cravo }\end{array}$ & $\begin{array}{c}\text { Cristiano } \\
\text { Moraes } \\
\text { Bilacchi } \\
\text { Azarias }\end{array}$ & $\begin{array}{l}\text { Esaú } \\
\text { Sirius } \\
\text { Ventura } \\
\text { Pupo }\end{array}$ & $\begin{array}{c}\text { Fernanda } \\
\text { Dantas } \\
\text { Bueno }\end{array}$ & $\begin{array}{l}\text { Gustavo } \\
\text { Brito de } \\
\text { Azevedo }\end{array}$ & $\begin{array}{l}\text { Louise } \\
\text { Catheryn } \\
\text { e Barne }\end{array}$ & $\begin{array}{l}\text { Mateus } \\
\text { Silvestrin }\end{array}$ & $\begin{array}{c}\text { Raphael } \\
\text { Y. de } \\
\text { Camarg } \\
\text { o }\end{array}$ & $\begin{array}{c}\text { Raymundo } \\
\text { Machado de } \\
\text { Azevedo } \\
\text { Neto }\end{array}$ & $\begin{array}{c}\text { Renan } \\
\text { Schiavoli } \\
\text { n Recio }\end{array}$ & $\begin{array}{c}\text { Vanessa } \\
\text { Carneiro } \\
\text { Morita }\end{array}$ \\
\hline $\begin{array}{c}\text { Conceptualiz } \\
\text { ation }\end{array}$ & $\mathrm{x}$ & $x$ & $x$ & $x$ & $x$ & $x$ & $x$ & & $x$ & $x$ & $x$ \\
\hline Methodology & $x$ & $x$ & $x$ & $x$ & $x$ & $x$ & $x$ & & $x$ & $x$ & $x$ \\
\hline Software & & & & $x$ & & & & & & $x$ & \\
\hline Validation & & & $x$ & & $x$ & $x$ & $x$ & & $x$ & & \\
\hline $\begin{array}{l}\text { Formal } \\
\text { Analysis }\end{array}$ & $x$ & $x$ & $\mathrm{x}$ & & $x$ & $x$ & $x$ & $x$ & & & $\mathrm{x}$ \\
\hline \multicolumn{12}{|l|}{ Investigation } \\
\hline Resources & $x$ & & & & $x$ & & & & $x$ & & \\
\hline $\begin{array}{l}\text { Data } \\
\text { Curation }\end{array}$ & $x$ & $x$ & $x$ & & $x$ & $x$ & $x$ & & $x$ & & $\mathrm{x}$ \\
\hline $\begin{array}{l}\text { Writing - } \\
\text { Original } \\
\text { Draft } \\
\text { Preparation }\end{array}$ & $x$ & $x$ & $x$ & & $x$ & $x$ & $x$ & & $x$ & & \\
\hline $\begin{array}{l}\text { Writing - } \\
\text { Review \& } \\
\text { Editing }\end{array}$ & $x$ & $x$ & $x$ & $x$ & $x$ & $x$ & $x$ & $x$ & $x$ & & \\
\hline Visualization & $x$ & $x$ & $x$ & $x$ & & $\mathrm{x}$ & $x$ & & & & \\
\hline Supervision & $x$ & & & & & & & & $x$ & & \\
\hline $\begin{array}{c}\text { Project } \\
\text { Administrati } \\
\text { on }\end{array}$ & $x$ & & & & & & & & $x$ & & \\
\hline $\begin{array}{c}\text { Funding } \\
\text { Acquisition }\end{array}$ & $x$ & & & & & & & & $x$ & & \\
\hline
\end{tabular}

